PALEO

Revue d'archéologie préhistorique

$14 \mid 2002$

Varia

\title{
La redécouverte du nouveau-né néandertalien Le Moustier 2
}

The rediscovery of the Le Moustier 2 Neandertal neonate

Bruno Maureille

\section{(2) OpenEdition}

Journals

Édition électronique

URL : http://journals.openedition.org/paleo/1458

DOI : $10.4000 /$ paleo.1458

ISSN : 2101-0420

Éditeur

SAMRA

Édition imprimée

Date de publication : 1 décembre 2002

Pagination : 221-238

ISSN : $1145-3370$

\section{Référence électronique}

Bruno Maureille, «La redécouverte du nouveau-né néandertalien Le Moustier 2 », PALEO [En ligne], 14 | 2002, mis en ligne le 17 août 2010, consulté le 23 juillet 2020. URL : http://journals.openedition.org/ paleo/1458; DOI : https://doi.org/10.4000/paleo.1458

Ce document a été généré automatiquement le 23 juillet 2020.

\section{(c) (i) (9)}

PALEO est mis à disposition selon les termes de la licence Creative Commons Attribution - Pas d'Utilisation Commerciale - Pas de Modification 4.0 International. 


\section{La redécouverte du nouveau-né néandertalien Le Moustier 2}

The rediscovery of the Le Moustier 2 Neandertal neonate

Bruno Maureille

Je tiens à exprimer ma gratitude à Jean-Jacques Cleyet-Merle, Directeur du Musée National de Préhistoire, qui m'a confié la fouille et l'étude des vestiges du Moustier 2 ainsi qu'aux Professeurs B. Vandermeersch et A. Mann pour leur soutien permanent. Lors de mes séjours au laboratoire du Musée National de Préhistoire, j'ai toujours été accueilli avec amitié. Je remercie sincèrement toutes les personnes qui y travaillent et particulièrement J. Angot-Westin, Ch. Fortin, A.-M. Gadioux, Ph. Jugie, S. Madeleine, A. Morala et A. Turq. Je remercie aussi J.-M. Geneste, J.-Cl. Ramos, L. Casalis, Ph. Roux et R. Rousset pour leurs aides diverses. Je tiens à exprimer ma reconnaissance à $H$. Rougier et $P$. Murail pour nos discussions et leur constante disponibilité.

Introduction : les vestiges humains de l'abri inférieur du Moustier, inventaire et devenir 
Fig. 1 - Localisation des sites du Moustier (abris inférieur et supérieur). Carte M. Seurin, UMR 5809 CNRS

Fig. 1 - Localisation of the Le Moustier prehistoric sites (upper and lower rock-shelters). Map M. Seurin, UMR 5809 CNRS

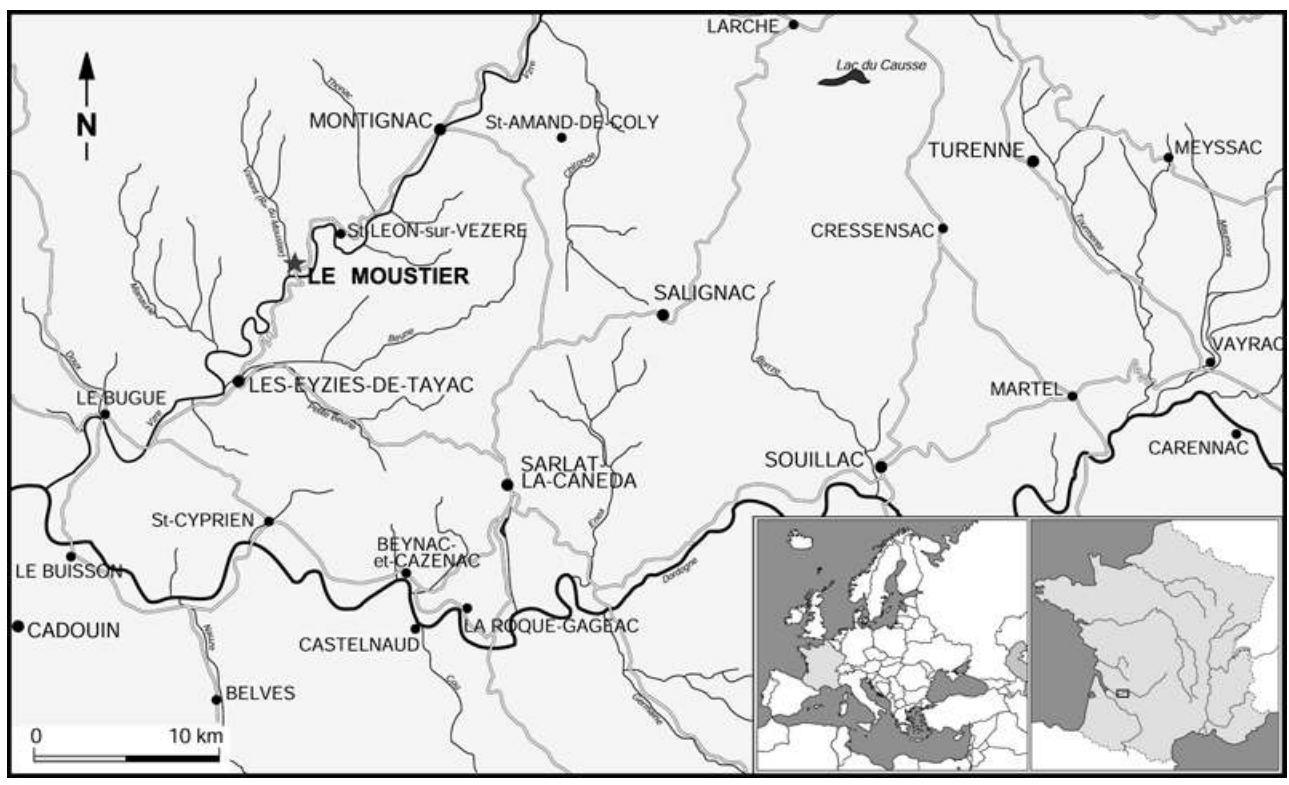

1 Nombreux sont ceux qui connaissent le destin du Néandertalien Le Moustier 1 (Hauser 1909 ; Vandermeersch 1971). Son squelette découvert par O. Hauser en 1908 fut partiellement détruit à la fin de la seconde guerre mondiale lors de l'incendie du Museum für Völkerkunde de Berlin (Heberer 1957 ; Vallois 1957). La tête osseuse, ayant été isolée et stockée séparément, fut sauvée (Müller 1965-1966 ; Vallois 1967). Si Hauser (1909) considérait que le niveau ayant livré le Moustier 1 se rapportait à l'Acheuléen, Bordes (1959) pensait que ce fossile était associé à la couche J (cf. infra).

Trois autres individus ont été mis au jour dans l'abri inférieur du Moustier :

1. un premier squelette fut découvert en 1896 à son extrémité ouest. Il pourrait être numéroté Le Moustier 4. Son contexte archéologique n'est pas très précis ainsi que sa localisation exacte. A l'époque, la majorité de la communauté scientifique ne considère pas ce spécimen comme un fossile du Paléolithique (voir Rivière 1906, 1909 ; Rutot 1908, 1910). A partir de la description préliminaire faite par $\mathrm{E}$. Rivière et des photos publiées de la tête osseuse (Rivière 1911 pl. I et II), nous sommes sûr qu'elle est moderne et gracile. On ne sait plus où se trouvent ces ossements.

2. en juillet 1910, O. Hauser découvre un fragment crânien et problablement à la même période, une dent (Drößler 1988; Vallois et Movius 1953, p. 149 note 1). Les données sur le contexte archéologique de ces vestiges sont toujours inédites. Ces restes pourraient être inventoriés comme Le Moustier 3 (Rosendhal, soumis) et Le Moustier 3 bis. Leur localisation actuelle est inconnue (Rosendahl ibidem).

3. en mai 1913, à la suite d'un effondrement d'une partie de la stratigraphie, D. Peyrony fait des fouilles dans l'abri inférieur, à l'ouest de celles de O. Hauser. Le 19 mai 1914, il découvre une fosse qui contient des restes humains. Ils sont identifiés comme Le Moustier 2 (Vandermeersch 1971).

3 Les informations fiables sur Le Moustier 2 et son contexte archéologique sont sommaires (Peyrony 1921 p. 4-5, 1930 p. 33-34) :

- la fosse est creusée à partir du niveau archéologique $\mathrm{J}$, traverse le niveau I et entame le niveau H. Ses limites sont claires ; 
- elle a la forme d'un cône tronqué de $40 \mathrm{~cm}$ de profondeur avec une section ovalaire en surface et un diamètre de $50 \mathrm{~cm}$;

- à l'intérieur, le mélange des sédiments des couches J, I et H est évident. La compacité de ces derniers est équivalente à celle des couches archéologiques ;

- la fosse contient le squelette d'un très jeune enfant ou d'un fœtus ;

- divers objets moustériens sont trouvés à côté des restes humains ;

- aucun matériel préhistorique (ni historique) plus récent n'est présent.

Mais D. Peyrony ne donne aucune information sur la position des restes humains à l'intérieur de la fosse, ni sur la façon dont il les a prélevés. La seule représentation des vestiges du Moustier 2 dans leur fosse, sera fournie par F. Bordes en 1972. Mais ce dessin ne semble pas basé sur des informations inédites qu'il aurait possédées. Si Vandermeersch (1971) signale que la localisation du Moustier 2 est inconnue, J.-L. Heim (1976, p. 6, note 1) considère que : «ce très jeune squelette, découvert par Hauser et signalé dans les notes manuscrites de D. Peyrony que nous avons consultées au Musée des Eyzies, a été très certainement égaré après avoir été envoyé pour étude à Marcellin Boule ». Les travaux plus récents, par exemple May (1986), Defleur (1993), Otte (1993), n'apportent aucune précision supplémentaire sur la nature de ces vestiges et leur devenir. Seule Binant (1991) fait exception. Elle mentionne, sans que l'on puisse savoir d'où proviennent ces informations, que l'état de conservation du Moustier 2 est bon et que le squelette postcrânien a été détruit en 1945 sans avoir été étudié.

Durant l'été 1996, avec l'équipe du MNP, après un inventaire synthétique des fossiles présents dans la collection anthropologique, une recherche de restes humains inédits fut entreprise dans les réserves. Avec le matériel lithique des abris du Moustier, des restes humains furent «isolés». Depuis le début des années 1990, ils avaient été « retrouvés » et étaient connus des membres de l'équipe du Musée (Cleyet-Merle, com. pers.) Ils étaient rassemblés dans un portoir sur lequel était mentionné « squelette ». A l'intérieur du portoir, quelques os étaient isolés avec des éclats moustériens, d'autres ossements humains étaient englobés dans des mottes de sédiments peu volumineuses. Enfin, d'autres mottes contenaient des ossements partiellement visibles sans que l'on puisse affirmer leur nature humaine. Un premier examen des pièces isolées montra :

1. qu'elles étaient celles d'un nouveau-né,

2. que le NMI était de un,

3. que l'état de conservation, la couleur, etc, plaidaient pour leur appartenance à un même individu. D'ailleurs des "collages» furent vite trouvés ${ }^{1}$. L'hypothèse que ces vestiges puissent appartenir au Moustier 2 étant évidente, il devenait nécessaire de prouver qu'il s'agissait bien des restes mis au jour par D. Peyrony et qu'ils étaient ceux d'un Néandertalien.

\section{Rappels sur les fouilles, la stratigraphie et la chronologie de l'abri inférieur du Moustier}

Peu de scientifiques ont fouillé l'abri inférieur du Moustier

7 - O. Hauser, à partir de 1907 et jusqu'en 1910, dont la qualité des travaux archéologiques est très variable. Il ne distingue que trois couches : la supérieure livrant du matériel remanié, un niveau sableux stérile sus-jacent à une couche acheuléenne qui 
livre la sépulture. Il n'y a pas eu d'étude plus précise du matériel archéologique mis au jour par ce fouilleur.

8 - D. Peyrony en 1912, 1913, 1914 et quelque temps en 1930 y travaille pour établir la stratigraphie et définir les cultures préhistoriques présentes. Ses résultats ont fait l'objet d'une monographie (Peyrony 1930) et sont rappelés dans le tableau 1.

9 - En 1937 et 1947, pour certains niveaux, M. Bourgon donna des attributions chronologiques différentes de celles de D. Peyrony. M. Bourgon (1957, p. 21) est le premier à proposer de nommer ce site " abri Peyrony ».

10 - F. Bordes a étudié le matériel lithique mis au jour (Bordes 1948). Puis, associé avec E. Bonifay, ils restaurèrent en 1961 les coupes endommagées à la suite d'une inondation (Laville et al. 1980, p. 173). Selon J.-Ph. Rigaud (com. pers.), ils travaillèrent surtout sur la partie basse de la stratigraphie. C'est avec le matériel de la couche J que F. Bordes et $M$. Bourgon testèrent pour la première fois le diagramme cumulatif (Bordes in Bourgon 1957). Enfin, F. Bordes présenta une révision du matériel lithique provenant de l'abri inférieur (Bordes 1961, 1969). Tous ces travaux précisèrent l'évolution des cultures lithiques établie par D. Peyrony et modifièrent leur attribution chronologique (tab. 1).

11 - H. Laville et J.-Ph. Rigaud, en 1969, « rafraîchirent » les profils en vue de la visite des membres de l'excursion A5 du VIIIe congrès de l'INQUA. Cela permit une nouvelle analyse granulométrique et sédimentologique des couches, souligna l'existence d'une surface d'érosion au sommet de la couche $G$ et modifia la corrélation chronoenvironnementale des niveaux archéologiques (tab. 1 et 2).

12 - En 1982, J.-M. Geneste et J.-P. Chadelle recueillirent des silex chauffés pour obtenir des datations absolues par thermoluminescence. Les résultats furent présentés dans deux publications (Valladas et al. 1986, 1987). En 1991, une seconde méthode a été utilisée (la résonance paramagnétique électronique, RPE ou Electron Spin Resonance, ESR). Cette technique donne des dates plus récentes que la précédente (tab. 2).

13 - Enfin, en 1992, les coupes ont été préparées pour les protéger par des murs en pierres. Ceux-ci furent terminés rapidement et des moulages des profils furent disposés pour présenter la stratigraphie du gisement (1994). Même si les collections disponibles peuvent faire l'objet de nouvelles recherches (par exemple Soressi 1999), cette protection rend très difficile toute nouvelle recherche sur la stratigraphie de l'abri Peyrony. 
Tabl. 1 - Données publiées sur les niveaux archéologico-sédimentologiques définis à l'abri inférieur du Moustier : de O. Hauser (1909) à F. Bordes (1948)

Table 1 - Published data about the archaeologico-sedimentological levels from the Le Moustier inferior rock-shelter : from O. Hauser (1909) to F. Bordes (1948)

\begin{tabular}{|c|c|c|c|c|c|c|c|c|c|c|}
\hline \multicolumn{4}{|c|}{ D'apres Hauser (1909) } & \multicolumn{4}{|c|}{ D'apreses D. Peyrony (1930) } & \multirow{2}{*}{\begin{tabular}{|l|} 
'aarres \\
M. Bourgon (1937) \\
$\begin{array}{l}\text { Attribution } \\
\text { chronologique }\end{array}$ \\
\end{tabular}} & \multirow{2}{*}{$\begin{array}{l}\text { D'apres } \\
\text { M. Aourgon (1947) } \\
\text { Attributition } \\
\text { chronologique } \\
\end{array}$} & \multirow{2}{*}{$\begin{array}{l}\text { Dapris } \\
\text { F. Bordes (1948) } \\
\begin{array}{l}\text { Asssemblage } \\
\text { litique }\end{array} \\
\end{array}$} \\
\hline $\begin{array}{l}\text { Numero : } \\
\text { de couche }\end{array}$ & $\begin{array}{l}\text { Nature } \\
\text { sódimentologique }\end{array}$ & 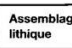 & \begin{tabular}{|l|}
$\begin{array}{l}\text { Numiero } \\
\text { de couche }\end{array}$ \\
\end{tabular} & $\begin{array}{l}\begin{array}{l}\text { Epaisseur } \\
\text { (mm) }\end{array} \\
\text { (m) }\end{array}$ & $\begin{array}{l}\text { Nature } \\
\text { sedimentologique }\end{array}$ & $\begin{array}{l}\text { Assemblage } \\
\text { lithique }\end{array}$ & $\begin{array}{l}\text { Attribution } \\
\text { chronologique }\end{array}$ & & & \\
\hline & \multirow{4}{*}{$\begin{array}{l}\text { Torreate } \\
\text { viegstale }\end{array}$} & \multirow{4}{*}{$\begin{array}{l}\text { Matérial } \\
\text { remanaié }\end{array}$} & & $?$ & Déblais & - & & & & \\
\hline & & & L & ${ }_{20}$ & $\begin{array}{l}\text { Sables cactareses } \\
\text { janurates }\end{array}$ & $\begin{array}{l}\text { Aurigracien } \\
\text { momen }\end{array}$ & & & & \\
\hline & & & k & 20 & $\begin{array}{l}\text { peu sableux } \\
\text { jaune terrevx }\end{array}$ & 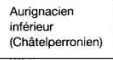 & & & & \\
\hline & & & s & 40 & Brun & $\begin{array}{l}\text { Moustéren } \\
\text { typoique }\end{array}$ & & Wừm & Wurm & $\begin{array}{l}\text { Moustefrien } \\
\text { typioue } \\
\text { tres peu de bitices) }\end{array}$ \\
\hline & Sables & Stêtile & I & 20 & $\begin{array}{l}\text { Sabes } \\
\text { fuviatiles }\end{array}$ & 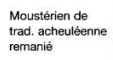 & 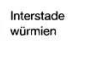 & Wừm & Würm & $?$ \\
\hline & \multirow{8}{*}{$\begin{array}{l}\text { Couche } \\
\text { archeoogique }\end{array}$} & \multirow{8}{*}{ Acheuléen } & н & $\begin{array}{l}120 \\
a_{130}\end{array}$ & $\begin{array}{l}\text { Brun au sommet } \\
\text { foyers base } \\
\text { chair al a base }\end{array}$ & $\begin{array}{l}\text { Moustitien de } \\
\text { trad achoubenne }\end{array}$ & Würm & Würm & Würm & $\begin{array}{l}\text { Moustérien de } \\
\text { trad achelferne } \\
\text { (peu de bifraces) }\end{array}$ \\
\hline & & & a & 60 & 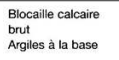 & $\begin{array}{l}\text { Moustarten de } \\
\text { trad acheulterne }\end{array}$ & Wum & wưrm & Würm & 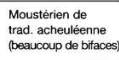 \\
\hline & & & $\mathrm{F}$ & $\begin{array}{l}10 \\
\text { al } \\
20\end{array}$ & $\begin{array}{l}\text { Argikse } \\
\text { fuvidiaes }\end{array}$ & $\begin{array}{l}\text { Moustricin de } \\
\text { trad acheuleenne }\end{array}$ & Wüm & & & 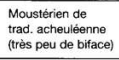 \\
\hline & & & E & 75 & 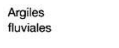 & Stérle & $\begin{array}{l}\text { Intergaciate } \\
\text { Ress Wurm }\end{array}$ & $:$ & & Stentile \\
\hline & & & D & 45 & $\begin{array}{l}\text { Giruiers } \\
\text { petitis } \\
\text { dilments }\end{array}$ & $\begin{array}{l}\text { Moustirien } \\
\text { remanien } \\
\text { peu niche }\end{array}$ & $\begin{array}{l}\text { Intergaciaire } \\
\text { Riss-Wümm }\end{array}$ & & & 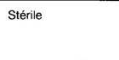 \\
\hline & & & c & 30 & $\begin{array}{l}\text { Graviers } \\
\text { gitemonts } \\
\text { moyens }\end{array}$ & $\begin{array}{l}\text { Mousterien } \\
\text { romanin } \\
\text { peur rehen }\end{array}$ & $\begin{array}{l}\text { Interglaciaire } \\
\text { Riss-Würm }\end{array}$ & & & Sterine \\
\hline & & & B & 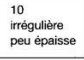 & 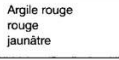 & 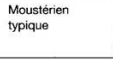 & $\begin{array}{l}\text { Integraciaire } \\
\text { Riss-Wirmm }\end{array}$ & 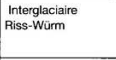 & Riss & 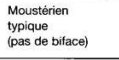 \\
\hline & & & A & 40 & 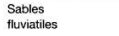 & Sterrile & $\begin{array}{l}\text { Integraciaire } \\
\text { Ress-Wurmm }\end{array}$ & & & \\
\hline
\end{tabular}

Tabl. 2 - Données publiées sur les niveaux archéologico-sédimentologiques définis à l'abri inférieur du Moustier : de F. Bordes (1969) à Mellars et Grün (1991)

Table 2 - Published data about the archaeologico-sedimentological levels from the Le Moustier inferior rock-shelter : from F. Bordes (1969) to Mellars and Grün (1991)

\begin{tabular}{|c|c|c|c|c|c|c|c|c|}
\hline \multicolumn{3}{|c|}{ D'apros F. Bordes (1969) } & \multicolumn{3}{|c|}{ D apres H. Laville et J.Ph. Rigaud (1973) } & \multirow{2}{*}{$\begin{array}{l}\text { D'apris Valladas of al. (1986) } \\
\text { Datations (TL) }\end{array}$} & \multirow{2}{*}{\multicolumn{2}{|c|}{ 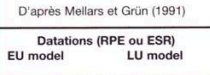 }} \\
\hline $\begin{array}{l}\text { Numiero } \\
\text { de couche }\end{array}$ & $\begin{array}{l}\text { Assemblage } \\
\text { lithique }\end{array}$ & $\begin{array}{l}\text { Attribution } \\
\text { chronologique }\end{array}$ & \begin{tabular}{|l|} 
Numéro \\
de couche
\end{tabular} & $\begin{array}{l}\text { Nature } \\
\text { sedimentologique }\end{array}$ & $\begin{array}{l}\text { Attribution } \\
\text { chronologique }\end{array}$ & & & \\
\hline L & Aurignacien I & Würm III & L & $\begin{array}{l}\text { Graviers et ébouilis emoussés } \\
\text { dans sable limaneux } \\
\text { peu argileux }\end{array}$ & & & & \\
\hline k & $\begin{array}{l}\text { Pérgordien I } \\
\text { Mousterien à denticulés }\end{array}$ & $\begin{array}{l}\text { Interstade } \\
\text { Wüm II. } \\
\text { Würm III }\end{array}$ & k & $\begin{array}{l}\text { Eboulis et graviers } \\
\text { alteres dans sabbe } \\
\text { argieux peu limoneux }\end{array}$ & & $42.000+1-3.200$ & & \\
\hline J & $\begin{array}{l}\text { Mousterien } \\
\text { typique }\end{array}$ & Würm II & $\mid \begin{array}{l}55 \\
J \\
31\end{array}$ & 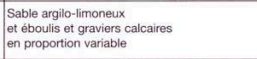 & $\begin{array}{l}\text { Würm II } \\
\text { porase IV } \\
\text { froid et sec }\end{array}$ & $40.300+/ 2.600$ & & \\
\hline 1 & $\begin{array}{l}\text { Moustétien } \\
\text { à denticulas }\end{array}$ & Würm II & 1 & $\begin{array}{l}\text { Sable argilo-limoneux } \\
\text { origignefluviatle } \\
\text { eboulis en enousses au sommet }\end{array}$ & $\begin{array}{l}\text { Wüm II } \\
\text { phases III } \\
\text { doux et humide }\end{array}$ & $40.900+1-5.000$ & & \\
\hline H & $\begin{array}{l}\text { Moustíren de } \\
\text { tratition acheuléenne } \\
\text { type B }\end{array}$ & Würm II & \begin{tabular}{|l}
$\mathrm{H} 9$ \\
$\mathrm{H}$ \\
$\mathrm{H} 1$
\end{tabular} & Formation hétérogène & $\begin{array}{l}\text { W-11: phase II } \\
\text { Würm II } \\
\text { W-11: phase I }\end{array}$ & $\begin{array}{l}\text { H9 а H2: } 42.500+/-2.000 \\
\text { H1: } 46.300+/-3.000\end{array}$ & $39.700+1-2.400$ & $.41 .000+1-2.600$ \\
\hline G & $\begin{array}{l}\text { Mousterien de } \\
\text { tration acheuléenne } \\
\text { type A Ace }\end{array}$ & Wüm II & \begin{tabular}{|l} 
G4 \\
G \\
G1
\end{tabular} & 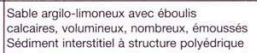 & $\begin{array}{l}\text { W-1: phase IX } \\
\text { W-1: phase VII }\end{array}$ & 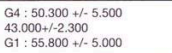 & $47.000+1-2.500$ & \\
\hline $\mathrm{F}$ & $\begin{array}{l}\text { Moustereien } \\
\text { a denticules }\end{array}$ & Würm II & F & $\begin{array}{l}\mid \begin{array}{l}\text { Limonon argilo-sableux } \\
\text { avec } \\
\text { quelques eboulis anguleux }\end{array}\end{array}$ & $\begin{array}{l}\text { Wurm I } \\
\text { phase VI } \\
\text { temperé }\end{array}$ & & & \\
\hline $\mathrm{E}$ & & $\begin{array}{l}\text { Interstade } \\
\text { Wüm I- } \\
\text { Würm II }\end{array}$ & E & $\begin{array}{l}\text { Limon dinondation } \\
\text { argileux et peu sableux }\end{array}$ & $\begin{array}{l}\text { Würm I } \\
\text { phase IV } \\
\text { temperér }\end{array}$ & & & \\
\hline D & & würm। & $\begin{array}{l}D_{2} \\
0 \\
01\end{array}$ & 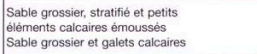 & $\begin{array}{l}\text { Wurm I } \\
\text { phase III } \\
\text { froid }\end{array}$ & & & \\
\hline c & & Wưrm 1 & c & $\begin{array}{l}\text { Formation fluviatle } \\
\text { Gaiets de quartizitit et rognonss de } \\
\text { silex roules adans sables grossiers }\end{array}$ & $\begin{array}{l}\text { Würm I } \\
\text { phase II } \\
\text { temperé et humide }\end{array}$ & & & \\
\hline B & $\begin{array}{l}\text { Moustetrien } \\
\text { typique }\end{array}$ & Wüm। & B & $\begin{array}{l}\text { Galets de quartizite et rognons de } \\
\text { silex roules dans sabies grossiers } \\
\text { Lentilles de limon argileux }\end{array}$ & $\begin{array}{l}\text { Würm I } \\
\text { phase II } \\
\text { temperé et humide }\end{array}$ & & & \\
\hline A & & Würm! & $\begin{array}{l}A_{2} \\
A \text { A1 }\end{array}$ & $\begin{array}{l}\text { Sable fin stratifié } \\
\text { Sable très grossier dans matrice argileuse }\end{array}$ & $\begin{array}{l}\text { Würm I } \\
\text { phase II } \\
\text { temperé et humide }\end{array}$ & & & \\
\hline
\end{tabular}




\section{Données inédites sur Le Moustier 2} de fouille et de restauration des vestiges du MNP, il était donc nécessaire de consulter des documents non publiés afin d'y trouver des informations inédites. Les archives de D. Peyrony conservées au Musée National de Préhistoire sont pauvres lorsque l'on considère son extraordinaire activité d'homme de terrain, les correspondances qu'il a entretenues. Mais, il tenait, plus ou moins régulièrement, un agenda où il mentionnait quelques-unes de ses activités. Ces écrits n'ont jamais été précisément étudiés dans la perspective d'une meilleure connaissance des évènements de la préhistoire périgourdine, des fouilles de D. Peyrony et d'autres préhistoriens.

C'est dans cet agenda que sont mentionnées les raisons qui poussent D. Peyrony à entreprendre des fouilles à l'abri inférieur. Il y est aussi signalé la date de la découverte de la sépulture du Moustier 2 (" elle contenait un petit squelette que nous avons attribué à un enfant ") et qu'il enverra à M. Boule "quelques objets » (fig. 2a). Etant donné la nature des échanges scientifiques entre D. Peyrony et M. Boule (cf. infra), nous supposons que par « objets» il faut comprendre « ossements ». Avec cet envoi, et comme nous l'assure la réponse de $\mathrm{M}$. Boule, $\mathrm{D}$. Peyrony souhaitait obtenir une estimation de l'âge au décès du spécimen. Malheureusement, il n'écrit pas ce qu'il a précisément soumis à M. Boule, ni s'il y a joint des informations sur le gisement et la stratigraphie. Cinq jours après, $M$. Boule a répondu en lui signalant que "les ossements" sont ceux d'un nouveau-né (fig. 2b). Ces deux passages de l'agenda rendent caduques l'hypothèse de J.-L. Heim (1976) selon laquelle Le Moustier 2 a été perdu à Paris. En effet, il est évident que le premier d'entre eux (fig. 2a) est celui cité par J.-L. Heim (1976, p. 6, note 1, cf. supra). Nous savions déjà que cette citation était en partie erronée, Le Moustier 2 n'ayant pas été mis au jour par O. Hauser. Selon nous, ils nous assurent aussi que D. Peyrony ne fit pas parvenir à M. Boule la totalité des vestiges du Moustier 2. En effet, il nous semble probable que par "quelques objets ", D. Peyrony voulait dire " quelques ossements » et non pas la totalité de la sépulture. Un fait va également dans ce sens. M. Boule publiait régulièrement des notes dans L'Anthropologie où il mentionnait les fossiles, les moulages, le matériel, venant enrichir les collections du laboratoire de Paléontologie du Muséum national d'Histoire naturelle (Boule 1915, 1923). Or, il n'a jamais écrit, dans une publication de ce type, que les restes du Moustier 2 lui aient été confiés. Il n'y avait donc aucune raison de supposer que ces ossements aient quitté le Périgord. 
Fig. 2a - Extrait de l'agenda de D. Peyrony mentionnant l'envoi à M. Boule d'ossements du Moustier 2.

Fig. 2a - Extract of Peyrony's journal mentioning the sending to M. Boule of some of the Le Moustier 2 bones.

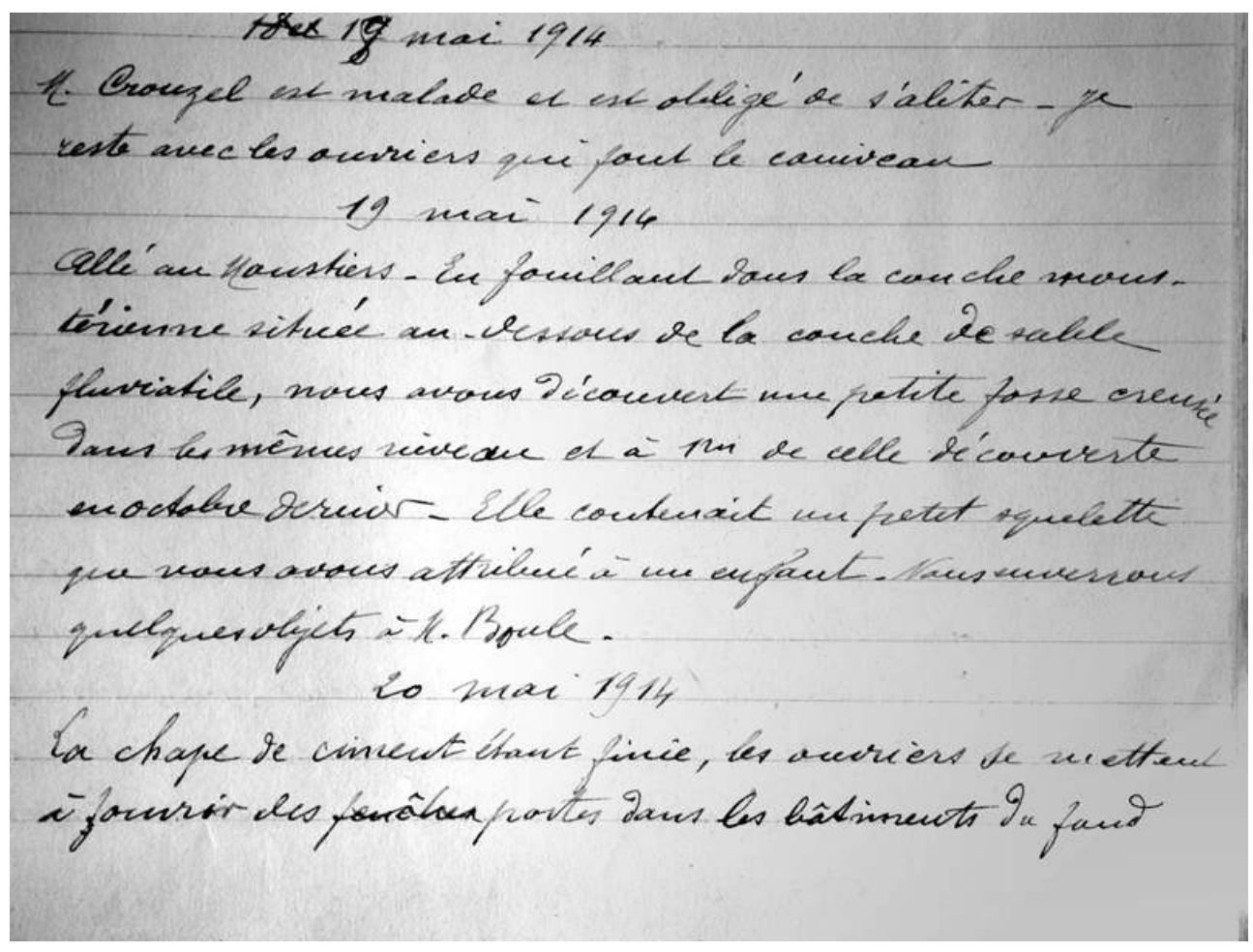

Archives Casalis, photo B. Maureille

Casalis archives, photo B. Maureille 
Fig. $2 b$ - Extrait de l'agenda de D. Peyrony mentionnant la réponse de $M$. Boule. Fig. $2 b$ - Extract of Peyrony's journal mentioning the answer of M. Boule.

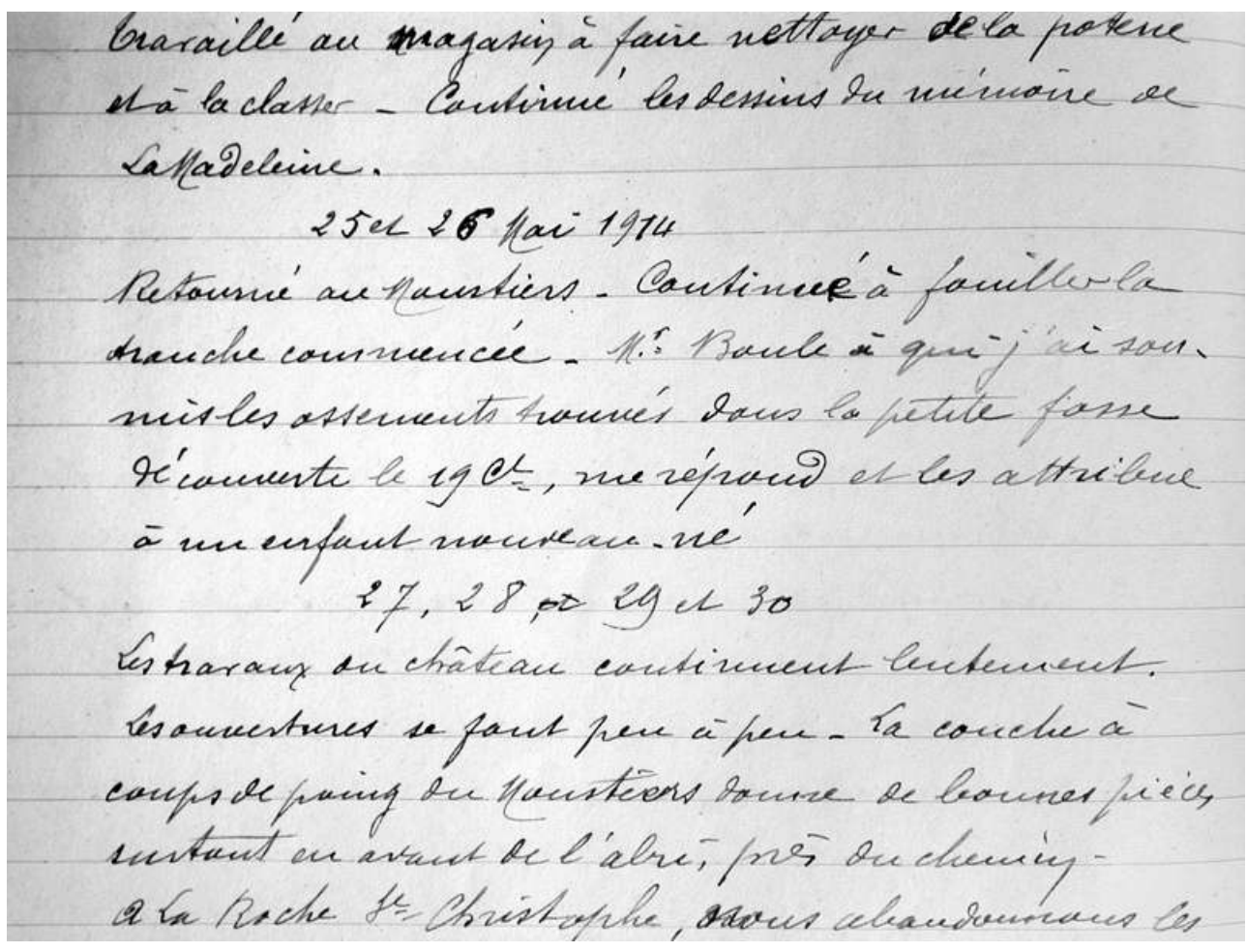

Archives Casalis, photo B. Maureille

Casalis archives, photo et @ B. Maureille

Malheureusement, au début du $\mathrm{XX}^{\text {ème }}$ siècle, les fossiles immatures intéressent assez peu la communauté scientifique et D. Peyrony lui même ne signale pas l'existence du Moustier 2 dans certaines de ses publications (par exemple Peyrony et Capitan 1924 ; Peyrony 1934b, 1949). Ce spécimen n'est pas mentionné dans le premier ouvrage sur les Hommes fossiles de M. Boule (1921). Sa découverte n'est pas plus citée dans ses commentaires critiques sur la monographie de D. Peyrony sur les abris du Moustier (Boule 1931). Seul, E. Hue signale, en 1937, que Le Moustier 2 est dans les collections du Musée de Préhistoire aux Eyzies. Mais, certaines des informations fournies dans ce catalogue sont erronées. Après la seconde guerre mondiale, on peut considérer que l'existence du Moustier 2 est oubliée. H.-V. Vallois ne le cite pas dans le premier vrai catalogue des hommes fossiles (Vallois et Movius 1953) ni dans l'inventaire des Néandertaliens de France (Vallois 1960). Enfin, nous avons déjà signalé ce que les membres de la communauté scientifique actuelle ont écrit sur le devenir de ce spécimen.

\section{Les restes humains du MNP, fouilles, matériel archéologique associé et sédiments.}

En avril 1997, la fouille des mottes de sédiment, contenant les vestiges osseux, put débuter ainsi que le nettoyage, la restauration des ossements épars. Au total, cela nécessita près de 150 jours de travail, avec parfois la nécessité de fouiller sous une loupe binoculaire, en humidifiant le sédiment à l'aide d'acétone. De nombreuses photographies noir et blanc, des diapositives en couleur (avec différents formats), des 
photographies numériques ont été réalisées ainsi que des relevés (échelle 2/1) des étapes de la fouille. Tous les objets furent coordonnés dans les trois dimensions de l'espace, le sédiment fut préservé ainsi que le moindre vestige lithique, la faune, les cailloutis calcaires.

Le sédiment est un sable argileux brun clair riche en muscovite. Ses caractéristiques minéralogiques (minéraux lourds) sont très proches de celles des couches $\mathrm{H}$, I et J de l'abri inférieur du Moustier et, plus largement, des alluvions de la Vézère. En revanche, elles différent nettement de celles des dépôts du grand abri de La Ferrassie (Texier, com. pers.) En ce qui concerne la texture, la courbe granulométrique cumulative est voisine de celle de la couche $\mathrm{J}$ mais aussi similaire à la courbe de la couche $\mathrm{M}$ de La Ferrassie (Texier, com. pers.)

19 Les mottes de sédiments ont aussi livré des produits de débitage d'un silex de couleur noire et peu de restes de faune. Tout le matériel lithique collecté s'accorde avec celui provenant de la partie supérieure (niveaux $\mathrm{H}, \mathrm{I}$ et J) de la séquence moustérienne de l'abri inférieur du Moustier aussi bien en ce qui concerne les aspects technologiques que la nature de la matière première (Morala et Turq, com. pers.) Il en est de même avec la faune: Rangifer tarandus, Cervus sp., Capra hircus ibex et un gros Bovinae sp. (Madeleine, com. pers.)

20 A la fin de cette première étape, nous sommes en présence d'un squelette bien représenté d'un nouveau-né (fig. 3). L'état de conservation des ossements est bon même si des éléments de la voûte crânienne (par exemple les pariétaux) sont brisés en une multitude de fragments. L'écaille de l'occipital est déformée et une côte gauche était réduite à l'état de fantôme. Les os sont de couleur claire. Ils sont fragiles et cassants. L'examen à la loupe binoculaire de leur surface montre des arêtes aiguës, non érodées. Ils ont donc été bien protégés par leur enfouissement et n'ont pas subi de processus érosifs. 
Fig. 3 - Etat de conservation du squelette du nouveau-né Le Moustier 2.

Fig. 3 - Preservation of the Le Moustier 2 perinate skeleton.

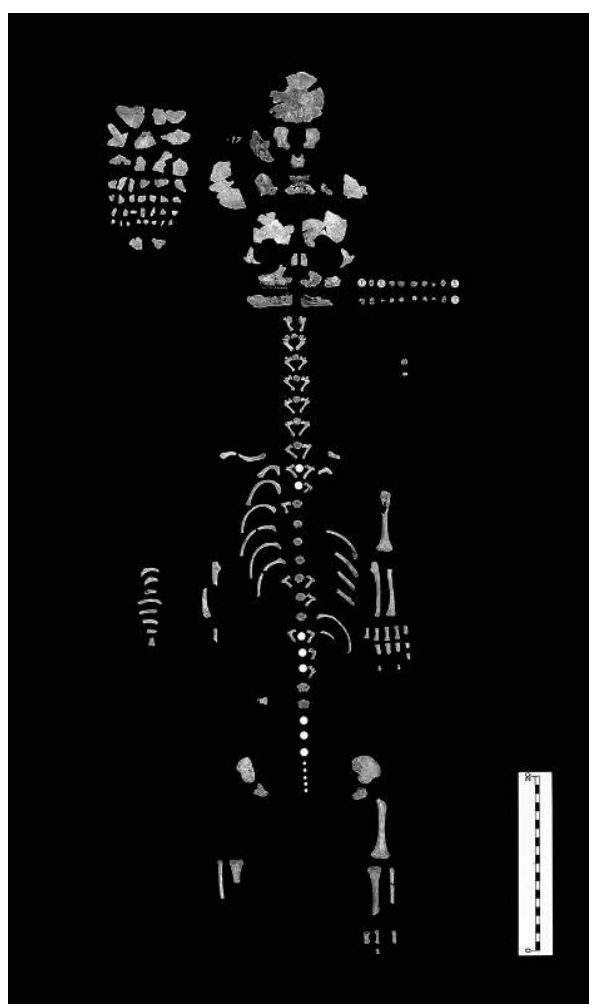

Notons que les deux ossements (l'humérus et le fémur droits) attribués à La Ferrassie 4 n'ont pas été associés à la reconstitution. L'élément représentant la partie latérale de la clavicule droite est en fait la moitié sternale de première côte gauche.

Note, that the two bones (right humerus and femur) misidentified as La Ferrassie 4 have not been associated to the presentation. The fragment representing the lateral part of the right clavicule is in fact the sternal half of the first left rib.

Photo Ph. Jugie, collection et (c) Musée National de Préhistoire

Photo Ph. Jugie, collection and (c) Musée National de Préhistoire

\section{Les vestiges du nouveau-né du MNP sont ceux du Moustier 2}

21 L'étape suivante de notre travail fut donc de démontrer que ces restes correspondaient à ceux du Moustier 2.

Trois éléments vont dans ce sens :

1. nous savons comment les collections du MNP ont été constituées (le Musée de Préhistoire des Eyzies deviendra le MNP en 1972 ; Cleyet-Merle et Marino-Thiault 1990). Il n'est pas possible que des mélanges aient eu lieu entre du matériel anthropologique provenant de différents sites, d'autant que les fossiles humains du MNP sont peu nombreux relativement au nombre de découvertes réalisées en Dordogne ;

2. les collections du Moustier proviennent presque exclusivement des fouilles de D. Peyrony. Les travaux plus récents (cf. supra) furent plus limités et aucun vestige humain n'y fut mis au jour ;

3. à la fin de la fouille des mottes de sédiments, nous sommes en présence d'un squelette très bien représenté (fig. 3). Seuls quelques "grands" ossements sont manquants: les deux scapulas, des côtes, l'humérus et le fémur droits. Des pièces plus petites sont aussi absentes : 
métacarpiens, métatarsiens, phalanges des deux côtés, corps de vertèbres et hémi-arcs neuraux. Mais, dans l'ensemble, les os longs présents et complets autant que l'humérus et le fémur droits du Moustier 2, existent. Il s'agit des deux seuls ossements représentant La Ferrassie 4. Rappelons que selon Heim (1976, 1982a, p. 8 et pl. I) ce spécimen est un fotus âgé, ou un nouveau-né. Or, cet auteur a supposé que ces deux ossements provenaient de la même fosse funéraire que celle de La Ferrassie 4bis (un nouveau-né néandertalien assez mal conservé) car il les retrouva avec les vestiges de ce fossile. Il a aussi souligné que la couleur des pièces osseuses de La Ferrassie 4bis différent fortement de celle des deux os de «La Ferrassie $4 »$ (Heim 1982a, p. 24). Cette remarque est également vraie si on compare la fossilisation et l'altération de surface des ossements de "La Ferrassie 4 » avec celles des pièces attribuées aux autres Néandertaliens de ce site (obs. pers.) Pour toute personne ayant une certaine expérience de la fouille de sépultures de nouveau-né, il est très difficile d'accepter l'hypothèse de la présence, dans une même fosse funéraire (la $\mathrm{n}^{\circ} 4$ de La Ferrassie), de deux individus avec des altérations taphonomiques si différentes. Or, les similitudes, tant sur la morphologie, la symétrie, les insertions musculaires, les caractères discrets que sur la métrique ainsi que celles concernant la fossilisation, la couleur et l'aspect de la surface externe des diaphyses entre les deux os de «La Ferrassie 4 » et l'humérus et le fémur gauches du Moustier 2, sont très importantes. Enfin, autre argument, sur la diaphyse de l'humérus et sur celle du fémur droits de très petites plages de sédiments sont conservées. Il est de couleur brun clair et on peut y observer la présence de muscovite. Le sédiment de la couche $C$ de La Ferrassie est aussi brun mais, en fonction de ce qui est collé sur les ossements de La Ferrassie 5 (par exemple), il est bien plus sombre que celui de la couche $\mathrm{J}$ du Moustier. De plus, la muscovite, très fréquente dans le sédiment accompagnant les vestiges du Moustier 2, est plus rare à La Ferrassie (Texier, com. pers.) Selon nous, le fémur et l'humérus droits que J.-L. Heim (ibidem) a désigné comme "La Ferrassie 4 ", appartiennent en fait au squelette du Moustier 2. Ces ossements font donc partie des " quelques objets » que D. Peyrony a envoyés à M. Boule pour la diagnose de l'âge du Moustier 2.

Comment cette confusion a-t-elle été possible ? Deux raisons peuvent être avancées :

1. la principale d'entre elles est que M. Boule n'a jamais publié son travail sur les restes humains de La Ferrassie même si cette étude «était sur le point de paraître quand la guerre a éclaté » (Boule 1921, p. 192). Rappelons que M. Boule avait reçu, de D. Peyrony, la sépulture $\mathrm{n}^{\circ} 4$ de la Ferrassie sous la forme d'un prélèvement (Peyrony 1934a, le bloc A selon Heim 1976). Ce dernier fut fouillé au laboratoire de Paléontologie du MNHN où les précédents vestiges humains de La Ferrassie avaient été restaurés (par exemple, le crâne de La Ferrassie 1 y fut reconstitué ; Boule 1921 p. 192). Or, il est certain que M. Boule pouvait distinguer les ossements de «La Ferrassie 4 » de ceux de la Ferrassie 4 bis s'ils avaient été présents dans ce bloc. Mais M. Boule n'y identifia qu'un seul individu: un nouveau-né, qu'il numérota La Ferrassie 4. Nous sommes certain de cela puisqu'en 1924, M. Boule trouvant, au Musée de Préhistoire des Eyzies, un talus droit humain avec les vestiges de faune du niveau C de La Ferrassie, le numérota La Ferrassie 7. Selon lui, cet ossement isolé représentait donc le septième sujet mis au jour dans ce gisement. Cela signifie que M. Boule n'avait identifié qu'un sujet par sépulture (numérotées de 1 à 6 ) trouvées par D. Peyrony.

2. la seconde raison est que les fossiles humains confiés à M. Boule étaient mal inventoriés. Ces lacunes au niveau des informations durèrent près de 50 ans puisque J.-L. Heim (1976 p. 29) souligne que les vestiges de La Ferrassie étaient rarement marqués. Ce dernier insiste aussi sur le fait que lorsqu'il entreprit son étude, de nombreux ossements de ce site avaient été mélangés. En fait, ils ne furent "officiellement enregistrés sur le catalogue des entrées du Laboratoire d'Anthropologie du Musée de l'Homme » qu'en 1953 (Heim 1976 p. 30). Cela signifie aussi que M. Boule n'avait pas « individualisé » précisément l'humérus et le fémur droits du Moustier 2. Mais on ne sait pas si D. Peyrony avait transmis à M. Boule les informations suffisantes pour que ce dernier puisse savoir d'où ces deux ossements provenaient, quel 
était leur contexte archéologique (cf. supra). M. Boule aurait donc mis ces deux pièces avec les vestiges des autres fossiles que D. Peyrony lui avait déjà fait parvenir. Plus d'un demisiècle après leur découverte, il était impossible de savoir que ces deux ossements ne provenaient pas de ce site. L'hypothèse de J.-L. Heim (1976) fut que M. Boule avait oublié, ou n'avait pas su identifier ces deux pièces, et J.-L. Heim les rapporta au site de La Ferrassie...

24 Une conséquence de cette découverte est qu'aucune sépulture double moustérienne attribuable aux Néandertaliens n'a été mise au jour. Enfin, à La Ferrassie, on doit s'interroger sur le nombre minimal d'individus moustériens. Selon que l'on accepte ou pas l'hypothèse de l'association du talus La Ferrassie 7 avec La Ferrassie 3 (Heim, 1976, 1982a et b), il peut être de 7 ou de 8. Mais d'autres regroupements entre des vestiges isolés et des sujets plus complets ont été faits par J.-L. Heim lorsqu'il étudia cette collection. Il semble donc nécessaire de refaire un travail de fond sur les fossiles humains de La Ferrassie, sur le nombre d'individus présents, sur leur état de conservation (avec des schémas adaptés), etc. Cela implique que l'on puisse accéder au matériel anthropologique et que la totalité des archives sur ce site, sur les fossiles moustériens, sur leur découverte et particulièrement les manuscrits de M. Boule, qui existent toujours, soient disponibles ${ }^{2}$.

\section{Le Moustier 2 : un nouveau-né néandertalien}

Etant sûr que les vestiges " redécouverts » dans les collections du MNP en 1996 sont ceux du Moustier 2 et, considérant le fait qu'il y avait probablement eu au moins une inhumation intrusive dans certains des niveaux paléolithiques (cf. Le Moustier 4), il est nécessaire de tenter de définir leur statut taxinomique et de préciser l'âge au décès du spécimen.

En ce qui concerne l'âge au décès, l'ossification des ossements, leurs dimensions, la calcification des germes dentaires, vont dans ce sens. Les estimations de la stature du Moustier 2 d'après la longueur maximale du radius, de celle de l'ulna et de celle du fémur, avec les nouvelles équations proposées par P. Sellier et al. (1997, en fonction des travaux de Fazekas et Kosa (1978) et relativement à la variabilité de sujets européens) sont respectivement de 52,05 cm, 51,70 cm et 52,49 cm. Ainsi, l'estimation de l'âge au décès est de $10,55,10,45$ et 10,67 mois lunaires ce qui correspond à une moyenne de $9,38 \pm 0,1$ mois. Cela confirme la détermination de l'âge par M. Boule (cf. supra) puis par J.-L. Heim (1976, 1982a) sur la base du fémur et de l'humérus droits. Selon la méthode de C. Moorrees et al. (1963) pour déterminer l'âge d'individus actuels sur la base du développement des germes des dents déciduales, Le Moustier 2 a un âge qui ne peut excéder quatre mois après la naissance.

En ce qui concerne la diagnose taxinomique, les données archéologiques fournies par D. Peyrony vont dans le sens de l'appartenance du Moustier 2 à la lignée néandertalienne. Or, en 1914, l'expérience de terrain de D. Peyrony est déjà importante. Il a mis au jour les restes de l'enfant du Pech-de-l'Azé, fouillé les sépultures de La Ferrassie 1, de La Ferrassie 2, prélevé après avoir partiellement mis au jour les vestiges humains de La Ferrassie 3 et La Ferrassie 4bis (nous continuerons à désigner ce spécimen avec ce numéro alors qu'il est évident qu'il doit être re-numéroté La Ferrassie 4) et fouillé trois fosses moustériennes (deux à La Ferrassie, une au Moustier). Selon D. Peyrony, la fosse funéraire du Moustier 2 était très clairement visible, creusée à partir de la base de la couche J. D’après un document inédit de D. Peyrony (archives Casalis), nous savons que 
dans la zone où il fouilla, les niveaux supérieurs de l'abri inférieur du Moustier avaient été enlevés probablement au XVIII ${ }^{e}$ siècle lors de la construction d'une maison d'habitation. La hauteur entre le sommet de la sépulture et le sommet des niveaux archéologiques devait être seulement de $25 \mathrm{~cm}$. Heureusement, D. Peyrony a noté qu'il n'y avait aucun matériel plus récent et/ou historique dans la fosse et que la composition et la compacité des sédiments de la fosse étaient identiques à celles des niveaux moustériens. Selon ce dernier, on ne peut douter que la sépulture soit creusée à partir d'un niveau moustérien. Or, pour le moment, en Europe de l'Ouest, les vestiges humains découverts en contexte moustérien sont tous rapportés à la lignée néandertalienne.

Enfin, les caractéristiques morphologiques du squelette sont différentes de celles des nouveau-nés actuels alors qu'elles sont proches de celles des Néandertaliens immatures et même adultes d'Europe et du Proche-Orient. Ainsi, Le Moustier 2 présente déjà un maxillaire sans dépression infra-orbitaire (comme elle est définie dans Maureille 1994). Sur sa face palatine, la suture prémaxillaire est ouverte avec antérieurement deux sinus interincisifs. Une telle combinaison de caractères est très rare chez les sujets immatures actuels et les premiers Hommes modernes alors qu'elle est typique des jeunes Néandertaliens comme Roc-de-Marsal, Pech-de-l'Azé et Dederiyeh burial 1 (Maureille et Bar 1999; Dodo et al. 2002). De plus, la suture prémaxillaire est aussi ouverte sur le plancher des fosses nasales et la totalité de la hauteur de la face nasale du processus frontal des maxillaires. Il a été démontré que cette morphologie est un caractère dérivé des jeunes Néandertaliens würmiens européens (Maureille et Bar ibid.) L'os zygomatique possède un corps assez court relativement à la hauteur de son processus frontal comme sur les Néandertaliens immatures Dederiyeh 1, Pech-de-l'Azé et La Quina H18 (Dodo et al. 1998) et les Néandertaliens adultes (Maureille 1994). La morphologie des os nasaux du Moustier 2 est particulière. Ils possèdent le même profil dans le plan sagittal que ceux des adultes néandertaliens avec un tiers proximal très vertical et les deux tiers distaux presque horizontaux (Trinkaus 1983; Maureille ibid.) D'autres traits plus subtils peuvent être observés sur les pars lateralis et basilaris de l'occipital et sur le pétreux. Sur les osselets de l'oreille moyenne, on peut noter des différences entre la morphologie néandertalienne et celle des Hommes actuels. Certaines sont discutées comme l'asymétrie des branches de l'étrier (Heim 1982a versus Arensburg et al. 1996) qui existe chez Le Moustier 2. Les couronnes des germes des incisives centrales et latérales déciduales sont en pelle et, pour la face vestibulaire, biconvexes. Ces traits sont fréquents chez les Néandertaliens (Patte 1962) et plus rares chez les hommes actuels. En ce qui concerne leurs dimensions, les diamètres coronaires des deux incisives déciduales supérieures du Moustier 2 se situent dans la partie inférieure de $95 \%$ de la variabilité néandertalienne. Pour les germes des incisives déciduales inférieures, les mêmes mensurations sont telles que Le Moustier 2 est exclu de la variabilité néandertalienne (et de celle de notre échantillon actuel). Des caractéristiques dérivées néandertaliennes peuvent être aussi déterminées sur le squelette post-crânien. C'est le cas de la courbure médio-latérale du radius. Pour ce trait, cet os est identique à ceux des enfants néandertaliens La Ferrassie 3 et La Ferrassie 4bis et distinct de ceux de Qafzeh 10 et Qafzeh 12 (Tillier 1999) et d'un large échantillon de nouveau-nés nubiens (obs. pers.) La proportion relative des phalanges du premier rayon de la main est en accord avec ce qui a été observé sur les mains de Néandertaliens adultes (Musgrave 1971; Heim 1982b ; Trinkaus, 1983 ; Vandermeersch 1991). L'orientation de l'extrémité proximale de l'ulna relativement à l'axe de la 
diaphyse (contra Hambucken 1993), la courbure moins prononcée de l'angle costal postérieur (Boule 1911-1913) sont des caractères assez distinctifs entre Néandertaliens et Hommes actuels. Même si cela ne représente pas des apomorphies néandertaliennes, l'épaisseur et la massivité des os (crâniens ou post-crâniens) doivent être soulignées. Aucune pathologie n'a pu être remarquée.

En fonction des données archéologiques et anthropologiques, il est donc certain que Le Moustier 2 est un nouveau-né néandertalien. Après La Ferrassie 5, il est le Néandertalien français le plus jeune mis au jour. C'est aussi un des nouveau-nés du Pléistocène parmi les mieux préservés (avec le sujet de Mezmaiskaya, Ovchinnikov et al. 2000). Il est aussi un des Néandertaliens les mieux conservés, y compris les adultes. Enfin, en acceptant la probable contemporanéité de la sépulture avec la couche J, Le Moustier 2 est un des Néandertaliens les plus récents d'Europe de l'Ouest.

\section{Pourquoi Le Moustier 2 est-il resté en Périgord?}

Malgré les archives il n'est pas possible de répondre avec certitude à cette question. Nous ne pouvons pas exclure que le début de la première guerre mondiale (la mobilisation en France fut déclarée le 1er août 1914) ne permit pas à D. Peyrony de faire parvenir à M. Boule la totalité des restes du Moustier 2. Mais, dans ce cas, D. Peyrony aurait pu compléter son envoi après cette guerre (il fut conservateur du Musée de Préhistoire des Eyzies de 1928 à 1936). Il ne le fit pas et jamais les vestiges du Moustier 2 ne quittèrent le Périgord. Ainsi, une autre hypothèse, présentant deux facettes distinctes, peut être avancée.

31 1) en décembre 1913, il y avait eu en Périgord un événement majeur pour la discipline. D. Peyrony avait eu l'assurance de la création du Musée de Préhistoire des Eyzies (ce dernier sera inauguré en septembre 1923; Cleyet-Merle et Marino-Thiault 1990). Il devait donc rassembler des collections concernant tous les champs de la Préhistoire. S'il disposait d'une grande quantité de matériel lithique, de vestiges de faune, de blocs gravés, de pièces d'art mobilier, provenant de ses fouilles, il avait très peu de fossiles humains. Les spécimens célèbres du Paléolithique supérieur tels que les sujets de CroMagnon, l'homme écrasé de Laugerie-Basse étaient " parisiens » depuis longtemps. Le Moustier 1 et l'homme de Combe-Capelle étaient en Allemagne. Le squelette du CapBlanc appartenait au commanditaire des fouilles de ce site. Concernant ses propres découvertes, les fossiles de La Ferrassie 1, 2, 3 et 4bis avaient déjà été envoyés au Muséum National d'Histoire Naturelle. La découverte du Moustier 2 intervenant en mai 1914, nous pouvons supposer que D. Peyrony ait tenu à conserver ce spécimen pour le futur musée des Eyzies. Selon nous, un fait va dans ce sens. D. Peyrony n'enverra plus de fossile humain au laboratoire de Paléontologie du Muséum national d'Histoire Naturelle après 1913. Ainsi, les restes de La Ferrassie 5 et 6, mis au jour en 1920 et 1921 par D. Peyrony et J.-L. Capitan, faisaient partie des collections du Musée des Eyzies en 1952 comme l'atteste leur localisation dans le catalogue des Hommes fossiles de Vallois et Movius (1953). Ils arrivèrent au Musée de l'Homme probablement en 1953 parce que H.-V. Vallois souhaitait achever le travail de M. Boule sur les restes humains de La Ferrassie. Alors, D. Peyrony n'était plus conservateur au Musée de Préhistoire des Eyzies depuis longtemps.

2) Denis Peyrony devait être déçu du traitement que $M$. Boule avait réservé à ses découvertes anthropologiques. Selon nous, D. Peyrony (1869-1954) et M. Boule 
(1861-1942) sont deux personnalités scientifiques différentes. Le premier, instituteur, est issu d'un milieu modeste (archives Casalis). Il abandonne sa profession d'Instituteur en 1910 en devenant chargé de mission au Ministère de l'Instruction publique et des Beaux-Arts. Il sera inspecteur des monuments préhistoriques et conservateur du Musée des Eyzies de 1928 à 1936, année où il prend sa retraite. M. Boule assez rapidement (à 23 ans) travaille avec d'importantes personnalités scientifiques parisiennes. A 41 ans, il est titulaire de la chaire de Paléontologie du MNHN. Il ne nous semble pas que D. Peyrony et M. Boule aient eu des relations amicales autres que celles qui existent entre deux

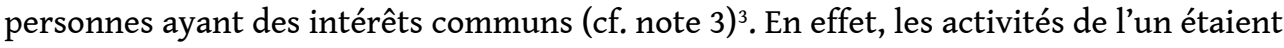
très utiles à l'autre et vice versa. Ainsi, le premier fouillait des sites périgourdins avec des aides financières du Ministère de l'Instruction publique et des Beaux Arts, le second était membre de la sous-commission des monuments mégalithiques (J.-L. Capitan en fut vice-président puis président) dépendant de ce ministère, commission qui attribuait des financements. Or, alors que M. Boule avait été capable de réaliser la monographie du fossile néandertalien de La Chapelle-aux-Saints en moins de 5 ans, en utilisant souvent des données provenant des adultes de La Ferrassie, il n'avait rien publié sur les spécimens, pourtant bien mieux conservés, de ce site. Une lettre inédite de D. Peyrony conservée dans les archives du Musée des Antiquités Nationales (MAN, Saint-Germainen-Laye) plaide en faveur de cette deuxième facette de notre hypothèse. Ainsi, en 1924 ou 1925, D. Peyrony avait proposé à M. Boule de publier dans les "Archives» la monographie de La Ferrassie et d'y inclure l'étude anthropologique. Ce dernier ne lui répondit jamais mais lui reprocha, au début des années 1930, de vouloir publier cette monographie ailleurs et sans lui. Il est vrai que cette publication commune aurait été assez difficile. M. Boule était un opposant farouche à l'hypothèse de l'existence de pratiques funéraires néandertaliennes alors que D. Peyrony, dès 1921, et sur la base de ses découvertes à La Ferrassie et au Moustier, avait écrit le premier article synthétique sur le sujet.

\section{Conclusions}

En septembre 1996, des vestiges humains appartenant à un nouveau-né sont exhumés des collections du Moustier du Musée National de Préhistoire. Certains ossements sont isolés, d'autres encore pris dans des mottes de sédiment. La lecture d'archives, certaines inédites, la fouille des blocs de sédiment, la restauration de certaines pièces, la reconstitution préliminaire du sujet (fig. 3), nous ont permis de prouver qu'il s'agit du Moustier 2, spécimen mis au jour par D. Peyrony le 19 mai 1914 et perdu pour la science depuis 88 ans.

Nous avons pu aussi confirmer les hypothèses proposées par D. Peyrony et M. Boule, à savoir que ces vestiges appartiennent à un Néandertalien (D. P.), qui plus est, nouveauné (M. B.) Une datation absolue directe (SMA) du fossile devrait être entreprise dans un avenir proche d'autant que la préservation du collagène des ossements parait excellente ( $\mathrm{H}$. Bocherens, com. pers.) Cela nous autorise à espérer de bonnes perspectives pour les recherches concernant l'ADN mitochondrial et les isotopes du Carbone et de l'Azote du collagène des os du spécimen.

La redécouverte du Moustier 2 a aussi permis de souligner que l'unique sépulture double néandertalienne n'existe plus, les deux ossements définissant La Ferrassie 4 appartenant au Moustier 2. 

entre Le Moustier 2 et un échantillon de nouveau-nés actuels ou sub-actuels (essentiellement des sujets européens et nubiens). Cela va dans le sens de l'hypothèse présentée récemment sur l'éloignement entre le patrimoine génétique des Néandertaliens et celui des Hommes actuels (Krings et al. 1997, 1999, 2000). Toutefois, il est nécessaire de rappeler que nous ne connaissons pas la variabilité morphologique des nouveau-nés du début du Paléolithique supérieur et que les vestiges plus anciens appartenant à cette classe d'âge et attribués au premiers Hommes modernes sont très (trop) fragmentaires (Tillier 1999). Nous n'en savons pas plus en ce qui concerne la variabilité génétique des Hommes fossiles modernes du Paléolithique moyen (ainsi que celle des spécimens du Paléolithique supérieur) alors qu'il a été souvent démontré qu'il existe un chevauchement de la variabilité métrique des ossements des premiers Hommes modernes avec celle des Néandertaliens (voir par exemple, Maureille et Houët 1998 ; Maureille et al. 2001).

La redécouverte du Moustier 2 est donc un événement important de la paléoanthropologie française (Maureille 2002). Elle augmente notre échantillon de Néandertaliens bien conservés, même si la classe d'âge du Moustier 2 rend moins impressionnant l'état de conservation de son squelette que celui d'un adulte. Son étude sera riche en données inédites. Elles seront incontournables dans le cadre de la réflexion sur l'ontogenèse des Néandertaliens, leur variabilité, ainsi que sur les relations phylogénétiques entre ces derniers et les Hommes modernes du Paléolithique moyen. Le Moustier 2 se classe parmi les très rares spécimens exceptionnels à intégrer dans notre réflexion sur l'évolution humaine et sa diversité.

\section{BIBLIOGRAPHIE}

ARENSBURG B., TILLIER A.-M., CHECH M. 1996 - The Subalyuk 2 middle ear stapes. Inter. Journ. Osteoarch., 1996, 6, p. 185-188.

BAR-YOSEF O. et VANDERMEERSCH B. (Eds) 1991 - Le squelette moustérien de Kébara 2. Paris : Ed. du CNRS, 1991, p. 197.

BINANT P. 1991 - Les sépultures du Paléolithique. Paris : Ed. Errance, 1991, p. 108.

BOCHERENS H., FIZET M., MARIOTTI A., LANGE-BADRE B., VANDERMEERSCH B., BORELl J.-P. et BELLON G. 1991 - Isotopic biogeochemistry $(13 \mathrm{C}, 15 \mathrm{~N})$ of fossil vertebrate collagen : application to the study of a past food web including Neandertal man. J. Hum. Evol., 1991, 20, p. 481-492.

BORDES F. 1948 - Les couches moustériennes du gisement du Moustier (Dordogne). Typologie et techniques de taille. Bull. Soc. Préhist. Fr, 1948, 45, p. 113-125. 
BORDES F. 1959 - Le contexte archéologique des Hommes du Moustier et de Spy. L'Anthropologie

(Paris), 1959, 63, p. 154-156.

BORDES F. 1961 - Mousterian Cultures in France. Science, 1961, 134, p. 803-810.

BORDES F. 1969 - Livret-guide de l'excursion A5. Landes - Périgord. Union Intern. Et. Quat., VIIIe Cong. INQUA., Paris, 1969, p. 56-62.

BORDES F. 1972 - A tale of two caves. New York : Haper \& Row, 1972, 169 p.

BOULE M. 1911-1913 - L'Homme fossile de La Chapelle-aux-Saints. Paris : Masson, 1913, p. 278.

BOULE M. 1915 - Nouvelles entrées dans les collections de Paléontologie du Muséum.

L'Anthropologie (Paris), 1915, 26, p. 182-183.

BOULE M. 1921 - Les hommes fossiles, éléments de Paléontologie. Paris : Masson \& Cie, 1921, p. 505.

BOULE M. 1923 - Nouvelles entrées dans les collections de Paléontologie du Muséum.

L'Anthropologie (Paris), 1923, 33, p. 280-281.

BOULE M. 1931 - Peyrony (D.) Le Moustier. Ses gisements, ses industries, ses couches géologiques. Rev. Anthrop., 1-6, 1930, 50 p. avec 19 fig. L'Anthropologie (Paris), 1931, 41, p. 161-164.

BOURGON M. 1937 - Contribution à l'étude du Quaternaire en Périgord noir. Bull. Soc. Hist. Archéol. Périgord, 1937, 64, p. 102-110, 165-173, 251-257.

BOURGON M. 1947 - Les anciens dépôts quaternaires du Périgord noir. Bull. Soc. Hist. Archéol. Périgord, 1947, 74, p. 52-62.

BOURGON M. 1957 - Les industries moustériennes et pré-moustériennes en Périgord. Arch. Inst. Pal. Hum., mém. 27. Paris : Masson et Cie, 1957, p. 141.

CLEYET-MERLE J.-J. et MARINO-THIAULT M.-H. 1990 - Les fouilles de l'état et le dépôt-musée des Eyzies. In : Lartet, Breuil, Peyrony et les autres... Une histoire de la préhistoire en Aquitaine. Sarlat : Min. Culture et SAMRA, p. 67-70.

DEFLEUR A. 1993 - Les sépultures moustériennes. Paris : CNRS éditions, 1993, p. 325.

DODO Y., KONDO O., MUHESEN S. et AKASAWA T. - 1998. Anatomy of the Neandertal infant skeleton from Dederiyeh cave, Syria. In : T. Akasawa, K. Aoki et O. Bar-Yosef (Eds), Neandertals and Modern Humans in Western Asia. Plenum Press, New York, 1998, p. 323-338.

DODO Y., KONDO O. et NARA T. - 2002. The skull of the Neanderthal child of burial $n^{\circ} 1$. In : T. Akasawa, S. Muhesen (Eds), Neanderthals burials. Excavations of the Dederiyeh cave, Afrin, Syria, Intern. Res. Cent. Japanese Studies, Kyoto, 2002, p. 93-137.

DROßLER R. 1988 - Flucht aus Dem Paradies. Halle-Leipzig : Mitteldeutscher Verlag, 1988, p. 384.

FAZEKAS I. G. et KOSA F. 1978 - Forensic Fetal Osteology. Akademiai Kiado, Budapest, 1978, p. 414.

HAMBUCKEN A. 1993 (inédit) - Variabilité morphologique et métrique de l'humérus, du radius et de l'ulna des Néandertaliens. Comparaison avec l'Homme moderne. Thèse Université Bordeaux 1, spécialité Anthropologie, nºrdre 903, 1993, p. 301.

HAUSER O. 1909 - Découverte d'un squelette du type du Néandertal sous l'abri inférieur du Moustier. L'Homme Préhistorique, 1909, 7, p. 1-9.

HEBERER G. 1957 - Bericht uber die bergung der skelettreste von Combe Capelle und Le Moustier aus dem brandschutt des berliner museums fur vor- und frühgeschichten Bericht $S$. Tagung de Deutschen gesellschaft für Anthropologie, 1956, p. 67-72.

HEIM J.-L. 1976 - Les hommes fossiles de La Ferrassie. I. Paris : Masson, 1976, p. 3-8. 
HEIM J.-L. 1982a - Les enfants néandertaliens de La Ferrassie. Paris : Masson, 1982, p. 169.

HEIM J.-L. 1982b - Les Hommes fossiles de La Ferrassie. Les squelettes adultes (squelettes des membres). Tome II. Archives Instit. Pal. Hum., mém. 38. Paris : Masson, 1982, p. 272.

HEIM J.-L. 1989 - L'apport de l'ontogénèse à la phylogénèse des Néandertaliens. In : G. Giacobini (éd.), Hominidae. Actes du 2ème congrès international de Paléontologie humaine. Turin, 28 Septembre - 3 Octobre 1987. Milan : Jaca Book, 1989, p. 339-346.

HUE E. 1937 - Crânes paléolithiques. Paris : A. Costes, 1937, p. 295.

KRINGS M., STONE A., SCHMITZ R. W., KRAINITZKI H., STONEKING M. et PAABO S. 1997 Neandertal DNA sequences and the origin of modern humans Cell, 1997, 20, p. 1-20.

KRINGS M., GEISERT H., SCHMITZ R. W., KRAINITZKI H., et PAABO S. 1999 - DNA sequence of the mitochodrial hypervariable region II from the Neandertal type specimen. Proc. Natl. Acad. Sci. USA, 1999, 96, p. 5581-5585.

KRINGS M., CAPELLI C., TSCHENTSCHER F., GEISERT H., MEYER S., HAESELER von A., GROSSSCHMIDT K., POSSNERT G., PAUNOVIC M. et PAABO S. 2000 - A view of Neandertal genetic diversity. Nature genetics, 2000, 26, p. 144-146.

LARTET E. et CHRISTY H. 1864 - Cavernes du Périgord. Objets gravés et sculptés des temps préhistoriques dans l'Europe occidentale. Paris : Lib. Académique, 1864, p. 8-9.

LARTET E. et CHRISTY H. 1875 - Reliquiae Aquitanicae. Being contributions to the Archaeology and Paleontology of Périgord and the adjoining provinces of Southern France. London : T. Rupert ed., 1875, p. 187 et pl.

LAVILLE H., RIGAUD J.-Ph. et SACKETT J. 1980 - Rock Shelters of the Perigord. New York : Academic Press, 1980, p. 173-178.

MADRE-DUPOUY M. 1992 - L'enfant du Roc-de-Marsal. Etude analytique et comparative. Paris : éd. du CNRS, 1992, p. 299.

MAUREILLE B. 1994 (inédit) - La face chez Homo erectus et Homo sapiens. Recherche sur la variabilité morphologique et métrique. Thèse de l'Université Bordeaux 1, t. 1 et t. 2, 1994, p. 486 et p. 148.

MAUREILLE B. 2002 - Lost Neanderthal neonate found. Nature, 2002, 419, p. 33-34.

MAUREILLE B. et HOUET F. 1998 - La variabilité morpho-métrique du nez : dérive génique dans la lignée néandertalienne ? Biométrie humaine et Anthropologie, 1998, t. 16, p. 27-34.

MAUREILLE B. et BAR D. 1999 - The premaxilla in Neanderthal and early modern children : ontogeny and morphology. Journal of Human Evolution, 1999, 37, p. 137-152.

MAUREILLE B. et SORESSI M. 2000 - A propos de la position chronostratigraphique de l'enfant du Pech-de-l'Azé (commune de Carsac, Dordogne) : la résurrection du fantôme. Paléo, 2000, 12, p. 339-352.

MAUREILLE B., ROUGIER H., HOUET F. et VANDERMEERSCH B. 2001 - Les dents inférieures du Néandertalien Regourdou 1 (commune de Montignac, Dordogne) : analyses métriques et comparatives. Paléo, 2001, 13, p. 183-200.

MAUREILLE B. et TURQ A. soumis - Excavations at the Le Moustier sites and their importance in French archaeology. In : H. Ullrich (Ed), The Neanderthal Adolescent Le Moustier 1 - New aspects, new results. Berlin : Museum für Vor- und Frühgeschichte.

MAY F. 1986 - Les sépultures préhistoriques. Paris : éd. du CNRS, 1986, p. 264. 
MELLARS P. et GRUN R. 1991 - A comparison of the electron spin rsonance and thermoluminescence dating methods : the results of ESR dating at Le Moustier (France). Cambridge Archaeol. Journ., 1991, 1, p. 269-276.

MOORREES C. F. A., FANNING E. A. et HUNT E. E., 1963 - Formation and resorption of three deciduous teeth in children. Am. J. Phys. Anthrop., 1963, 21, p. 205-213.

MULLER W. 1965-1966 - Der schädel des Homo mousteriensis Hauseri wieder in Berlin. Praehistorische Zeitschrift, 1965-1966, 63 \& 64, p. 2.

MUSGRAVE J. H. 1971 - How dextrous was Neanderthal man ? Nature, 1971, 233, p. 538-541.

OTTE M. 1993 - Préhistoire des religions. Paris : Masson, 1993, p. 140.

OVCHINNIKOV I. V., GOTHERSTROM A., ROMANOVA G. P., KHARITONOV V.M., LIDEN K. et GOODWIN W. 2000 - Molecular analysis of Neanderthal DNA from the northern Caucasus. Nature, 2000,404, p. 490-493.

PATTE E. 1962 - La dentition des Néandertaliens. Paris : Masson et Cie, 1962, p. 162.

PEYRONY D. 1921 - Les Moustériens inhumaient-ils leurs morts ? Périgueux : Ribes et Cie, 1921, p. 1-8.

PEYRONY D. 1930 - Le Moustier, ses gisements, ses industries, ses couches géologiques. Revue d'Anthropologie, 1930, 40, p. 48-76 et 155-176.

PEYRONY D. 1934a - La Ferrassie, Moustérien, Périgordien, Aurignacien. Paris : Lib. E. Leroux, 1934, p. 92.

PEYRONY D. 1934b - Guide illustré du savant et du touriste : Les Eyzies et les environs. Ussel : Imp. G. Eyboulet et fils, 1934, p. 32.

PEYRONY D. 1949 - Le Périgord préhistorique. Essai de géorgaphie humaine suivi des listes des stations, gisements, monuments divers connus, avec leur bibliographie. Périgueux : Soc. Hist. Archéol. Périgord, 19949, p. $22-23$ et 36.

PEYRONY D. et CAPITAN J.-L. 1924 - L'Humanité primitive dans la région des Eyzies. Paris : Stock, 1924, p. 126.

PONCE de LEON M. S. et ZOLLIKOFER C. P. E. 2001 - Neanderthal cranial ontogeny and its implications for late hominid diversity. Nature, 2001, 412, p. 534-538.

RIVIERE E. 1906 - Le squelette humain du Moustier (Dordogne). In : Cong. Préhist. de France. C.-R. première session - Périgueux 1905. Paris : Schleicher frères, 1906, p. 488-489.

RIVIERE E. 1909 - De l'antiquité paléolithique du squelette humain du Moustier-de-Peyzac (Dordogne). Bull. Soc. Préhist. Fr., 1909, 4, p. 142-143.

RIVIERE E. 1911 - La mandibule du squelette chelléo-moustérien de la femme du Moustier (Dordogne). In : Cong. Préhist. de France. C.-R. sixième session - Tours 1910. Paris : Bur. Soc. Préhist. Fr., 1911, p. 116-124.

ROSENDAHL W. (soumis) - Le Moustier 3 : a second Le Moustier Neanderthal discovery by Otto Hauser. In : H. Ullrich (Ed), The Neanderthal Adolescent Le Moustier 1 - New aspects, new results. Berlin : Museum für Vor- und Frühgeschichte.

RUTOT A. 1908 - Sur la découverte d'un squelette humain au Moustier (Vézère). Bull. Soc. Belge Géol., 1908, 22, p. 317-319.

RUTOT A. 1910 - Sur les nouvelles trouvailles de squelettes humains quaternaires dans le Périgord. Bull. Soc. Belge Géol., 1910, 24, p. 370-377. 
SELLIER P., TILLIER A.-M. et BRUZEK J. 1997 - A la recherche d'une référence pour l'estimation de l'âge des foetus, nouveau-nés et nourrissons des populations archéologiques européennes. Anthropologie et Préhistoire, 1997, 108, p. 75-87.

SORESSI M. 1999 - Variabilité technologique au Moustérien. Analyse comparée du débitage levallois MTA A du Moustier (Dordogne, France). Paléo, 11, p. 111-134.

TILLIER A.-M. 1983 - Le crâne de l'enfant d'Engis 2 : un exemple de distribution des caractères juvéniles, primitifs et néandertaliens. Bull. Soc. roy. Belge Anthrop. Préhist., 1983, 94, p. 51-75.

TILLIER A.-M. 1986a - Quelques aspects de l'ontogenèse du squelette crânien des Néanderthaliens In : V. V. Novotny et A. Mizerova A. (Eds), Fossil man, new facts, new ideas. Anthropos (Brno), 1986, 23, p. 207-216.

TILLIER A.-M. 1986b - Ordre d'apparition des caractères néandertaliens sur le squelette crânien au cours de la croissance. Problèmes phylogéniques. In : M. Sakka (éd.), Définition et origine de l'homme. Coll. Intern. CNRS n 3. Paris : éd. du CNRS, 1986, p. 263-270.

TILLIER A.-M. 1987 - L'enfant de La Quina H18 et l'ontogénie des Néandertaliens In : Préhistoire de Poitou-Charentes, problèmes actuels. Paris : éd. du CTHS, 1987, p. 201-206.

TILLIER A.-M. 1988 - A propos de séquences phylogénique et ontogénique chez les Néanderthaliens In : E. Trinkaus (Ed.), L'homme de Néandertal : l'anatomie., vol. 3. Liège : ERAULT, 1988, p. 125-135.

TILLIER A.-M. 1999 - Les enfants moustériens de Qafzeh. Interprétation phylogénétique et paléoauxologique. Cahiers de Paléoanthropologie. Paris : CNRS editions, 1999, p. 239.

TILLIER A.-M. 2000 - Palaeoauxology applied to Neanderthals. Similarites and contrasts between Neanderthal and Modern Human Children. Anthropologie (Brno), 2000, 38, p. 109-120.

TRINKAUS E. 1983 - The Shanidar Neandertals. New York : Academic Press, 1983, p. 502.

VALLADAS H., GENESTE J.-M., JORON J.-L. et CHADELLE J.-P. 1986 - Thermoluminescence dating of Le Moustier (Dordogne, France). Nature, 1986, 322, p. 452-454.

VALLADAS H., GENESTE J.-M., JORON J.-L. et CHADELLE J.-P. 1987 - Datations par la thermoluminescence de gisements moustériens du Sud de la France. L'Anthropologie (Paris), 1987, 91, p. 211-226.

VALLOIS H.-V. 1957 - Les squelettes du Moustier et de Combe-Capelle. L'Anthropologie (Paris), 1957, 61 , p. 569-570.

VALLOIS H.-V. 1960 - Répertoire des Hommes de Néandertal en France. Z. Morph. Anthrop., 1960, 50, p. $125-135$.

VALLOIS H.-V. 1967 - La réapparition du crâne de Moustier. L'Anthropologie (Paris), 1967, t. 71, p. 191.

VALLOIS H.-V. et MOVIUS Jr. H. L. 1953 - Catalogue des Hommes fossiles. In : C. R. XIXe session Cong. Géol. Intern., commission pour l'Homme fossile, section $V$ : les préhominiens et les hommes fossiles, fasc. V, Alger 1952. Macon : Presses Imp. Protat frères, 1953, p. 122-167.

VANDERMEERSCH B. 1969 - Les nouveaux squelettes moustériens découverts à Qafzeh (Israël) et leur signification. C. R. Acad. Sc. Paris, 1969, sér. D, t. 268, p. 2562-2565.

VANDERMEERSCH B. 1971 - France : Le Moustier. In : K. Oakley, B. G. Campbell. et T. Molleson (Eds), Catalogue of fossil Hominids, part II : Europe, London : Trustees of the British Mus. (Nat. Hist.), 1971, p. 149-150. 
VANDERMEERSCH B. 1981 - Les Hommes fossiles de Qafzeh (Israël), Paris : Ed. du CNRS, 1981, p. 319.

VANDERMEERSCH B. 1991 - La ceinture scapulaire et les membres supérieurs. In : O. Bar-Yosef et B. Vandermeersch (Eds), Le squelette moustérien de Kébara 2. Paris : Ed. du CNRS, 1991, p. 157-178.

\section{ANNEXES}

\section{An abridged English version}

Over the past one hundred and fifty years, excavations in the Department of the Dordogne (Southwest France) have produced a corpus of data which forms the basis of our reconstruction of Upper Pleistocene human evolution. Within this region, discoveries in the rock-shelters facing the Vézère river valley in the small village of Le Moustier (fig. 1), were amongst the most revealing of early sources of information about the biology and culture of Neandertals (Maureille and Turq, submitted).

Excavations in 1863-1864 by E. Lartet and H. Christy $(1864,1875)$ in the Le Moustier upper rock-shelter yielded stone tools that have come to be known as the Mousterian Industrial complex, a tool kit associated in Europe with Neandertals. Situated below this upper shelter is the lower (or Peyrony) rock-shelter where the remains of two hominid skeletons have been found associated with Mousterian tools.

\section{The humans remains from the Le Moustier inferior rock-shelter}

In 1908, O. Hauser (1909), a Swiss antiquity dealer, discovered the nearly complete skeleton of an adolescent Neandertal (Le Moustier 1), a specimen that was sold to the Berlin museum of Ethnology and was damaged during World War II.

On 19 May 1914, during excavations along the western edge of the earlier Hauser explorations, D. Peyrony $(1921,1930)$ discovered the remains of another human skeleton. This discovery is listed as Le Moustier 2 in the catalogue of European human fossils (Vandermeersch 1971). According to Peyrony (1930; see also Laville et al. 1980) the Le Moustier 2 burial pit originated in archaeological level J (a $40 \mathrm{~cm}$ brown layer containing a Typical Mousterian industry; tab. 1) and was intrusive into levels I (a 20 $\mathrm{cm}$ sterile fluviatile level with Denticulate Mousterian tools heavily damaged by cryoturbation) and $\mathrm{H}$ (a 120-130 cm very rich brown layer, containing a Mousterian of Acheulian Tradition industry, type B). Archaeological levels $\mathrm{J}$ and $\mathrm{H}$ have been recently dated back (TL method), respectively, to 40,300 $\pm 2,600$ and 42,500 $\pm 2,000 \mathrm{BP}$ (Valladas $e t$ al. 1986; tab. 2). The burial pit has the shape of a truncated cone of $40 \mathrm{~cm}$ depth with an upper oval diameter of $50 \mathrm{~cm}$. The mixing of the sediments from layers J, I and $\mathrm{H}$ inside the pit as well as its limits were evident (Peyrony 1921, 1930). D. Peyrony recorded no data about the position of the skeleton in the pit nor any description of the state of conservation of the bones. It appears likely that immediately after the discovery, D. Peyrony " showed » some pieces of the skeleton to M. Boule, who was at the time examining fossils from earlier excavations of J.-L. Capitan and D. Peyrony at the La Ferrassie site. It is unclear, however, if M. Boule was given any information about the archaeological context of the infant skeleton and the Le Moustier site. Peyrony's diary 
mentions that five days after the discovery of Le Moustier 2, M. Boule informed him that this was the skeleton of a neonate.

Although D. Peyrony mentioned the Le Moustier 2 discovery in his publications about the site and discussions about Mousterian funeral practises, the specimen was not mentioned by M. Boule in his often highly critical appraisal of D. Peyrony's interpretations of the site's geological formation (Boule 1931). More important, it was also unlisted in the published listing of the human fossil finds from the Périgord " sent » to the Paleontological Department of the Musée National d'Histoire Naturel (MNHN) in Paris (Boule 1923) and was not mentioned in M. Boule synthetic books on human evolution (Boule 1921). In his 1936 catalogue of Paleolithic skulls, E. Hue noted that the Le Moustier 2 specimen was located at the Musée de Préhistoire des Eyzies (Hue 1937). But after the death of D. Peyrony (the $26^{\text {th }}$ of November 1954), the existence of this fossil, or even its location, was no longer acknowledged. Some scholars suggested that it had been lost in Paris (Heim 1976, p. 6, note 1 and fig. 2a). Others believed that its whereabouts were unknown (Vandermeersch 1971).

\section{The rediscovery of the Le Moustier 2 specimen}

In September 1996, in collaboration with members of the Musée National de Préhistoire (MNP) team, a comprehensive survey of the human remains in the archaeological collections of the Museum was undertaken. During this work, the remains of a human neonatal skeleton were found with the Le Moustier lithic collections. They were in a drawer containing Mousterian artifacts and some of the bones were isolated while others remained embedded in blocks of sediment. The bones were not marked nor was the container in which they were stored identified in any way. The possibility that this unidentified skeleton might be that of the missing Mousterian age infant mentioned by D. Peyrony led to an intensive examination of his unpublished notes and diaries concerning his work at the Le Moustier site (fig. 2a et $2 b$ ). These revealed no evidence that the remains of the Le Moustier 2 infant had at any time left the museum of Les Eyzies. Thus, the published suggestion that this skeleton had disappeared in Paris was mistaken.

\section{The hominid remains and associated archaeological artefacts}

Work began in April 1997 to remove the human bones from the blocks of sediment. The sediment is a clear brown-beige clayey sand. It contains numerous green hornblendes, garnets and mica particles of muscovite type. This leads to think that its depositional environment has been influenced by the Vézère river (Texier, pers. com.) Preliminary analyses reveal many similarities with the sedimentological constitution of the layers I and $\mathrm{J}$ of the Le Moustier inferior rock-shelter (Texier, pers. com.) The blocks also contained flint flakes and numerous flint chips. These stone artefacts are similar in raw material and in lithic technology with the artefacts found in levels $\mathrm{H}, \mathrm{I}$ or $\mathrm{J}$ at the Le Moustier site (Morala and Turq, pers. com.) Similarly, the faunal remains recovered from these blocks, mainly Rangifer tarandus, Cervus sp., Capra hircus ibex and a large Bovinae, represent the same species which are known from the same Mousterian levels (Madelaine, pers. com.) 
The excavation of the blocks of sediment (and associated works: pictures, drawings,...), which required around 150 days of work to complete, resulted in the recovery of a very well preserved neonate skeleton (fig. 3). The light brown to beige coloured bones are very fragile but their preservation is exceptional. Both parietals and the posterior part of the hemi-frontals, however, are broken into small fragments and will be very difficult to reconstruct.

Comparison of the stages of dental calcification of the preserved deciduous teeth with modern reference standards (Moorrees et al. 1963), suggests that the Le Moustier 2 individual was probably not more than four months of age when it died. Examination of the development of the cranial and post-cranial bones also suggests an age of death of around birth. Estimations of stature, based on the maximal diaphysal lengths of the radius, ulna and femur in human neonates (Fazekas and Kosa 1978; Sellier et al. 1997)are respectively $52,05 \mathrm{~cm}, 51,70 \mathrm{~cm}$ and $52,49 \mathrm{~cm}$. For neonates of living peoples, these stature estimates correspond to an age around birth (10,55, 10,45 and 10,67 lunar months, with a mean of 9,38 \pm 0.1 calendar months). This is in agreement with the M. Boule age at death estimation of the Le Moustier 2 specimen.

In addition to the many similarities in the composition of the sediments and the associated faunal and archaeological materials between these blocks and the Mousterian levels of the Le Moustier site, there are several other facts that also support the attribution of this skeleton to a Middle Palaeolithic cultural horizon. First, there is a detailed record in the MNP of their archaeological collections and it is highly unlikely that materials from different Périgord sites have been confused. Secondly, although there have been a number of excavations in the Le Moustier sites over the years, led by different investigators, the materials from this site in the MNP are only from D. Peyrony's field activities. Finally, as attested to by D. Peyrony in some of his unpublished writings, the skeleton of a single neonate was discovered and excavated from Mousterian levels.

\section{The misattribution of two bones: La Ferrassie 4 doesn't exist}

Except for both scapulae and pubis bones, the right femur and humerus and some phalanxes, metacarpals and metatarsals of both sides, all the major post cranial bones are preserved and successfully removed. After preliminary examination of the bones, it becomes apparent that the missing right femur and humerus do, in fact, exist, but have been misattributed to an individual from the La Ferrassie site. Indeed, Heim (1976, 1982a) frequently underlined that the La Ferrassie 4 specimen is only represented by a right humerus and a right femur. As this individual was absent from the previous $\mathrm{M}$. Boule inventory of the humans remains from this site (see also Vallois and Movius 1953; Vallois 1960), Heim (ibidem) has supposed that these bones were coming from the same burial (the « bloc A ») than the La Ferrassie 4bis neonatal skeleton and represented another new-born individual. Indeed, it has been also frequently noted that the colour and fossilization of the two long bones of La Ferrassie 4 are totally different from those of La Ferrassie 4bis (Heim, ibidem; Maureille pers. obs.; Mann pers. obs.), a situation very difficult to understand, and to accept, for remains that are supposed to derive from the same burial pit. Comparing the state of fossilisation, the colour of the bones and above all, the morphology, symmetry, muscular insertions, discrete traits and measurements, there is no doubt that the right femur and humerus of La Ferrassie 4 are 
the missing long bones of Le Moustier 2. The sediment still adhering to the two bones of La Ferrassie 4 is a clear brown-beige clayey sand with muscovite like the sediment from the blocks associated with the Le Moustier 2 specimen. Its color is different from those of the Mousterian $\mathrm{C}$ level from the La Ferrassie site where muscovite is more rare than at the Le Moustier inferior rock-shelter (Texier, pers. com.). Considering these data, it is clear that these two bones were those given to M. Boule by D. Peyrony in May 1914 for the determination of the age at death of Le Moustier 2 and latter misattributed to the La Ferrassie hominid sample. A consequence of this discovery is that, in contrast to the double burial of early modern human from Middle Palaeolithic (Vandermeersch 1969, 1981), there is no longer any evidence for this practice in Western Europe Middle Palaeolithic times. Another consequence is that, at the La Ferrassie site, only 7 different individuals have been found (if we accept the association of the La Ferrassie $n^{\circ} 7$ isolated bone with the La Ferrassie $n^{\circ} 3$ individual; Heim 1982a): La Ferrassie $n^{\circ} 1, n^{\circ} 2$, $n^{\circ} 3, n^{\circ} 4$ (the ancient $n^{\circ} 4$ bis), $n^{\circ} 5, n^{\circ} 6$ and $n^{\circ} 8$. This shows clearly the necessity to work again on this fundamental fossil human collection to produce, at least, a new and unquestionable inventory of the individuals present in this site.

\section{The Le Moustier 2 specimen: a Neandertal neonate.}

It is also crucial to carefully examine the neonate skeleton to confirm its Neandertal status. In this context, it is useful to review D. Peyrony's long experience with the excavation of Middle Paleolithic sites. Prior to his work at Le Moustier, he had excavated the human remains associated with a Mousterian of Acheulean tradition (b type) cultural horizon at the Pech-de-l'Azé I site (Maureille and Soressi 2000)and uncovered a number of burials in the La Ferrassie site (Peyrony 1934a). He had also excavated three other pits with no human bones (two in the La Ferrassie site, one in the Le Moustier lower rock-shelter; Peyrony 1930, 1934a; Heim, 1976). It is clear from the excavation records that the Le Moustier 2 burial pit originated at the base of level J. Moreover, D. Peyrony also noted the total absence of more recent artefacts (i.e. from Upper Palaeolithic or Historic periods) around or inside the pit. He recorded also that the composition and compactness of its sediment were identical to that of the Palaeolithic levels (Peyrony 1930). For his part, D. Peyrony was certain that the burial derived from the Mousterian level $\mathrm{J}$ and was directly associated with a Middle Palaeolithic cultural tradition and no other scholar has ever doubted that this specimen could be more recent (for example see, Mellars and Grün 1991; Defleur 1993).

Perhaps even more compelling, the morphology of many parts of the skeleton exhibits a number of differences from equivalent structures of modern human new-born baby, but are very similar to the morphology of European and Near Eastern adult and juvenile Neandertals. For example, Le Moustier 2 has already a clear projecting maxilla. On its palatine face the premaxillary suture is fully opened with two interincisive sinuses. These traits are very very rare in anatomically modern humans and extant humans but are typical of Neandertals like Roc-de-Marsal or Pech-de-l'Azé (Maureille and Bar 1999). Moreover, the premaxillary suture is also opened on the nasal floor and the entire height of the nasal face of the frontal process of the maxilla. It has already been clearly demonstrated that this morphology is a very pertinent derived trait of the immature Wurmian Neandertals (Maureille and Bar ibid.). The zygomatic bone possesses a short body in comparison with the length of its frontal process, traits observed in immature Neandertals such as Dederiyeh 1, Pech-de-l'Azé and La Quina H18 
as well as being typical of adult individuals (Dodo et al. 1998; Maureille 1994). The morphology of the nasal bones is also distinctive. They possess the same sagittal profile found on adult Neandertals (Trinkaus 1983; Maureille ibid.) There are subtle traits distinctive of Neandertals on the lateral and basal portions of the occipital bone and the petrous portion of temporal bone. The asymmetry of the stapes arch is evident (Heim 1982a versus Arensburg et al. 1996). In the dentition, the crowns of the central and lateral upper deciduous incisors are shovel-shaped and bi-convex, features found in the Neandertal dentition but relatively uncommon in living Europeans. Metrically, the deciduous incisors are at the lowest end of the $95 \%$ interval of Neandertal variability for both the mesio-distal and bucco-lingal diameters. Many traits are also evident on the post-cranial skeleton as the medio-lateral curvature of the radius. Anatomically, the bone is identical to that of the Neandertal infants 3 and 4 bis from the La Ferrassie site and distinct from the Qafzeh one in Israel (Tillier 1999) and an archaeological sample of neonates from Nubia. The relative proportion of the thumb phalanxes (Musgrave 1971; Heim 1982b; Trinkaus, 1983; Vandermeersch 1991), the orientation of the ulna head (contra Hambucken 1993), the posterior costal angle which is less pronounced (Boule 1911-13) are also distinctive characters. Although not representing Neandertal derived traits, the robusticity and thickness of the cranial and post cranial bones are noteworthy.

Thus, there appear to be numerous morphological differences in many parts of the skeleton between Neandertal and modern human new-born baby as our preliminary analysis of the Le Moustier 2 remains reveals no evidence of pathology. Although this reinforces the hypothesis of major genetic differences between Neandertals and extant humans (Krings et al. 1997, 1999, 2000; Ovchinnikov et al. 2000), it is also necessary to keep in mind that the range of variation between neonates from European Middle Palaeolithic humans and Middle or Upper Palaeolithic anatomically modern people remains virtually unknown.

\section{Conclusions}

The evidence supports the rediscovery of the Le Moustier 2 human remains in the Musée National de Préhistoire collections and its identification as a Neandertal neonate. With the addition of the two long bones formerly attributed to the La Ferrassie 4 specimen and which are in fact two main long bones of the Le Moustier 2 individual, we have one of the most complete Neandertal immature specimen (fig. 3). Further, even among the entire Neandertal sample from the Wurm, Le Moustier 2 can be considered as one of the best preserved skeletons with the Roc-de-Marsal child (Madre-Dupouy 1992), the Kebara 2 individual (Bar-Yosef and Vandermeersch 1991) and the Dederiyeh burial 1 specimen (Dodo et al. 1998). Considering its age at death, it is next to the youngest Neandertal specimen from Western Europe (La Ferrassie 5) and preserves bones which were, until now, unknown in the Neandertal neonate sample as well as in the entire annals of Human Palaeontology. They will provide an important addition to the study of Neandertal ontogeny (Heim 1989; Tillier 1983, 1986a et b, 1987, 1988, 2000, Ponce de Léon and Zollikofer 2001). Preliminary tests seem to indicate that preservation of bone collagen appears excellent (Bocherens, pers. com.) Extraction of collagen samples will be planned for the recovery of DNA fragments as well carbon $\left({ }^{13} \mathrm{C} /{ }^{12} \mathrm{C}\right)$ and nitrogen $\left({ }^{15} \mathrm{~N} /{ }^{14} \mathrm{~N}\right)$ isotopic signatures (Bocherens et al. 1991$)$ as well as to obtain ${ }^{14} \mathrm{C}$ for the calculation, utilising accelerator mass spectrometry (AMS), of an 
absolute date of the individual. The rediscovery of the Le Moustier 2 Neandertal neonate after its almost 90 years disappearance (Maureille 2002) adds to our sample of this enigmatic fossil human and provides us with a fundamental piece in our ongoing attempt to understand the nature of human evolution.

\section{NOTES}

1. Un second examen des ossements du MNP, réalisé quelques mois plus tard par A.-M. Tillier (UMR 5809 CNRS), alla dans le même sens que nos premières investigations.

2. Selon J.-L. Heim (1976, p. 1), lorsqu'il commence son travail sur les spécimens de La Ferrassie, seules quelques notes manuscrites de M. Boule sur le crâne de La Ferrassie 1 et des os longs des adultes existent.

3. D. Peyrony ne « revendique » que trois longues amitiés au sein de la communauté scientifique française, celles avec J.-L. Capitan, H. Breuil et E. Cartailhac.

\section{RÉSUMÉS}

En 1996, des ossements d'un périnatal sont retrouvés dans les réserves du Musée National de Préhistoire avec les collections des abris du Moustier (Dordogne). Certains sont isolés, d'autres sont pris dans de petites mottes de sédiment. Assez vite, il apparaît que ces vestiges peuvent être ceux mis au jour par D. Peyrony dans l'abri inférieur. En 1997, le Musée National de Préhistoire nous confie la fouille des mottes et l'étude des vestiges. A l'issue de la fouille (fin 2000), nous proposons la reconstitution d'un squelette de périnatal exceptionnellement bien conservé. L'étude, morphologique et métrique, préliminaire, des vestiges osseux nous assure que nous sommes en présence d'un Néandertalien. Considérant sa classe d'âge, certains de ses ossements sont uniques dans les annales de la Paléontologie humaine. De surcroît, la fouille des mottes de sédiment s'est accompagnée de l'enregistrement de données qui permettront de préciser la position de segments du corps dans la tombe. Enfin, cette découverte remet en cause le nombre d'individus présents dans le gisement de La Ferrassie et l'existence de l'unique sépulture double du Moustérien d'Europe. En ce qui concerne les prédécesseurs des Hommes modernes d'Europe, Le Moustier 2 représente donc la plus importante découverte paléoanthropologique faite en France depuis ces vingt-cinq dernières années et en Périgord depuis 1961. Son étude permettra de nombreuses avancées en anthropologie biologique, sur l'ontogénie des Néandertaliens et les pratiques funéraires moustériennes.

We report here the rediscovery, in the archaeological collections of the Musée National de Préhistoire (Les Eyzies-de-Tayac), of a Neandertal neonatal skeleton from the Le Moustier inferior rock-shelter. This fossil has been excavated in 1914 but it was lost to science and unavailable for study until six years ago.

This specimen represents one of the best preserved Wurmian Neandertals, including adults. Considering the age at death of this individual (about four months postnatal) and the small number of previously discovered Neandertal neonatal specimens (only five individuals, some of them very fragmentary, since the beginning of the 20 th century), its study can provide significant data on Neandertal ontogeny. Many of the bones of the skeleton were previously 
unknown for human Palaeontology annals; further because of the precise techniques used to excavate the blocks of sediment containing the bones, it was possible to record information concerning the precise orientation of the skeleton in the burial pit. These observations can be employed in future research that focuses on Neandertal burial practices. It will also provide an additional context for the interpretation of Neandertal burial practices at the La Ferrassie site and for the constitution of the La Ferrassie human sample. Finally, the study of this skeleton will result in the accumulation of much basic biological data (e. g. ontogeny, and also the recognition of derived versus primitive traits) crucial to the ongoing discussions of the phylogenetic status and cultural capacities of the Neandertals. The Le Moustier 2 specimen is, for Middle Palaeolithic human remains, the most important discovery from France since 25 years and from Perigord since 40 years.

\section{INDEX}

Mots-clés : Néandertalien, Würm, Moustérien typique, périnatal, nouveau-né, sépulture, funéraire, Le Moustier, La Ferrassie

Keywords : Neandertal, Würm, Typical Mousterian, perinate, new-born, grave, burial, Le Moustier, La Ferrassie

\section{AUTEUR}

\section{BRUNO MAUREILLE}

UMR 5809 CNRS, Laboratoire d'Anthropologie des populations du passé, Université Bordeaux 1, avenue des Facultés, 33405 Talence cedex, France. Email : b.maureille@anthropologie.ubordeaux.fr 